\title{
CRESTED PRODUCTS OF ASSOCIATION SCHEMES
}

\author{
R. A. BAILEY AND PETER J. CAMERON
}

\begin{abstract}
In this paper, we define a new type of product of association schemes (and of the related objects, permutation groups and orthogonal block structures), which generalizes the direct and wreath products (which are referred to as "crossing" and "nesting" in the statistical literature.) Given two association schemes $\mathcal{Q}_{r}$ for $r=1$, 2, each having an inherent partition $F_{r}$ (that is, a partition whose equivalence relation is a union of adjacency relations in the association scheme), we define a product of the two schemes, which reduces to the direct product if $F_{1}=U_{1}$ or $F_{2}=E_{2}$, and to the wreath product if $F_{1}=E_{1}$ and $F_{2}=U_{2}$, where $E_{r}$ and $U_{r}$ are the relation of equality and the universal relation on $\mathcal{Q}_{r}$. We calculate the character table of the crested product, and show that if the two schemes $\mathcal{Q}_{1}$ and $\mathcal{Q}_{2}$ have formal duals, then so does their crested product (and we give a simple description of this dual). We make an analogous definition for permutation groups with intransitive normal subgroups, and show that the constructions for association schemes and permutation groups are related in a natural way.

The definition can be generalized to association schemes with families of inherent partitions, or permutation groups with families of intransitive normal subgroups. This time the correspondence is not so straightforward, and works as expected only if the inherent partitions (or orbit partitions) form a distributive lattice.

We conclude with some open problems.
\end{abstract}

\section{Introduction}

Following Bose and Shimamoto [8], we define an association scheme on a finite set $\Omega$ to be a partition of $\Omega \times \Omega$ into classes $\mathcal{C}_{i}$, for $i$ in $\mathcal{K}$, whose $(0,1)$ adjacency matrices $A_{i}$ are symmetric and satisfy

(i) there is a distinguished element 0 in $\mathcal{K}$ such that $A_{0}=I_{\Omega}$, the identity matrix on $\Omega$;

(ii) for all $i, j$ in $\mathcal{K}$, the product $A_{i} A_{j}$ is an integer linear combination of the $A_{k}$ for $k$ in $\mathcal{K}$.

It follows that $\sum_{i \in \mathcal{K}} A_{i}=J_{\Omega}$, the all-1 matrix on $\Omega$, and that the span $\mathcal{A}$ of $\left\{A_{i}: i \in \mathcal{K}\right\}$ over $\mathbb{R}$ is a commutative algebra, called the Bose-Mesner algebra of the scheme. The rank of the scheme is $|\mathcal{K}|$. Note that we do not follow Bannai $[6]$ in allowing non-symmetric adjacency matrices, apart from a remark at the end of Section 7.

The trivial association scheme on $\Omega$ has $A_{0}=I_{\Omega}$ and $A_{1}=J_{\Omega}-I_{\Omega}$. The isomorphism type of this association scheme is denoted by $\underline{\underline{n}}$, where $n=|\Omega|$.

Given two association schemes $\mathcal{Q}_{1}$ and $\mathcal{Q}_{2}$ on sets $\Omega_{1}$ and $\Omega_{2}$, with adjacency matrices $A_{i}\left(i \in \mathcal{K}_{1}\right)$ and $B_{j}\left(j \in \mathcal{K}_{2}\right)$, there are two well-established methods of combining them into a product association scheme on $\Omega_{1} \times \Omega_{2}$. The two methods were formalized by Nelder in a statistical context [15]. One is called crossing: it yields the direct product $\mathcal{Q}_{1} \times \mathcal{Q}_{2}$, whose adjacency matrices are

$$
A_{i} \otimes B_{j} \quad \text { for } i \text { in } \mathcal{K}_{1} \text { and } j \text { in } \mathcal{K}_{2} .
$$


The other is called nesting: it yields the wreath product $\mathcal{Q}_{1} / \mathcal{Q}_{2}$ (also denoted $\mathcal{Q}_{2}\left(\mathcal{Q}_{1}\right)$, whose adjacency matrices are

$$
A_{i} \otimes J_{\Omega_{2}} \quad \text { for } i \text { in } \mathcal{K}_{1} \backslash\{0\}
$$

and

$$
I_{\Omega_{1}} \otimes B_{j} \quad \text { for } j \text { in } \mathcal{K}_{2} .
$$

For example, $\underline{\underline{n}} \times \underline{\underline{m}}$ is the rectangular association scheme $\mathrm{R}(n, m)$; while $\underline{\underline{n}} / \underline{\underline{m}}$ is the group-divisible scheme $\operatorname{GD}(n, m)$ with $n$ blocks of size $m$.

The purpose of this paper is to give a single method of combining $\mathcal{Q}_{1}$ and $\mathcal{Q}_{2}$ into an association scheme on $\Omega_{1} \times \Omega_{2}$ that has both crossing and nesting as special cases. The new method can be described very naturally for the class of association schemes derived from orthogonal block structures. This is done in Section 2, where necessary information about orthogonal block structures is summarized. Orthogonal block structures are defined by partitions. Section 3 gives some results about partitions in general association schemes. These are used in Section 4 to extend the new product to general association schemes, using a distinguished partition in each scheme. Section 5 gives the character table of the crested product of two schemes, and Section 6 uses this to explore duality of crested products.

Direct and wreath products are also established ways of combining two permutation groups. Section 7 gives the crested product of permutation groups, in such a way that if each group preserves an association scheme then the crested product of the groups preserves the crested product of the schemes.

Finally, a more general version of the crested product is presented in Sections 810. Here a whole family of partitions is needed in each scheme, rather than just one. Pointers to further work are in Section 11.

\section{Orthogonal block structures}

An orthogonal block structure on a set $\Omega$ is a set of partitions of $\Omega$ satisfying some conditions. Orthogonal block structures were introduced in [17]. The following summary is based on [2].

Given a partition $F$ of $\Omega$, denote by $R_{F}$ the $\Omega \times \Omega$ relation matrix for $F$; that is $R_{F}(\alpha, \beta)=1$ if $\alpha$ and $\beta$ are in the same part of $F$, while $R_{F}(\alpha, \beta)=0$ otherwise. A partition is uniform if all its parts have the same size; if $F$ is uniform its part size is denoted by $k_{F}$. The two trivial partitions are the universal partition $U$, which has a single part, and the equality partition $E$, all of whose parts are singletons.

Partitions of $\Omega$ are partially ordered by the relation $\preccurlyeq$, where $F \preccurlyeq G$ if every part of $F$ is contained in a part of $G$. Given any two partitions $F$ and $G$, their infimum is the partition $F \wedge G$ whose parts are intersections of $F$-parts with $G$-parts. It is the coarsest partition which is finer than both $F$ and $G$. Their supremum is the partition $F \vee G$ whose parts are minimal subject to being unions of $F$-parts and unions of $G$-parts.

Definition. A set $\mathcal{F}$ of uniform partitions of $\Omega$ is an orthogonal block structure if

(i) $\mathcal{F}$ contains $U$ and $E$;

(ii) for all $F$ and $G$ in $\mathcal{F}, \mathcal{F}$ contains $F \wedge G$ and $F \vee G$;

(iii) for all $F$ and $G$ in $\mathcal{F}$, the matrices $R_{F}$ and $R_{G}$ commute with each other. 
Given a partition $F$ in an orthogonal block structure $\mathcal{F}$ on $\Omega$, define the adjacency matrix $A_{F}$ by

$$
A_{F}(\alpha, \beta)= \begin{cases}1 & \text { if } F=\bigwedge\left\{G \in \mathcal{F}: R_{G}(\alpha, \beta)=1\right\} \\ 0 & \text { otherwise. }\end{cases}
$$

It is shown in $[\mathbf{2}, \mathbf{1 7}]$ that $\left\{A_{F}: F \in \mathcal{F}, A_{F} \neq 0\right\}$ is an association scheme on $\Omega$.

Now let $F$ and $G$ be partitions of sets $\Omega_{1}$ and $\Omega_{2}$ respectively. Define $F \times G$ to be the partition of $\Omega_{1} \times \Omega_{2}$ whose relation matrix is $R_{F} \otimes R_{G}$. If $F$ and $G$ are both uniform then so is $F \times G$, and $k_{F \times G}=k_{F} \times k_{G}$.

Given orthogonal block structures $\mathcal{F}$ and $\mathcal{G}$ on $\Omega_{1}$ and $\Omega_{2}$ respectively, we can cross them to obtain $\mathcal{F} \times \mathcal{G}$ or nest them to obtain $\mathcal{F} / \mathcal{G}$. Here

$$
\mathcal{F} \times \mathcal{G}=\{F \times G: F \in \mathcal{F}, G \in \mathcal{G}\}
$$

while

$$
\mathcal{F} / \mathcal{G}=\left\{F \times U_{2}: F \in \mathcal{F}\right\} \cup\left\{E_{1} \times G: G \in \mathcal{G}\right\},
$$

where $E_{r}$ and $U_{r}$ are the trivial partitions of $\Omega_{r}$. It is shown in [17] that $\mathcal{F} \times \mathcal{G}$ and $\mathcal{F} / \mathcal{G}$ are both orthogonal block structures on $\Omega_{1} \times \Omega_{2}$. Furthermore, the operation of deriving the association scheme from the orthogonal block structure commutes with both crossing and nesting. Thus the notation $\underline{\underline{n}}$ can be used unambiguously for both the trivial association scheme on an $n$-set and the trivial orthogonal block structure $\{E, U\}$ on an $n$-set, while $\underline{\underline{n}} / \underline{\underline{m}}$ denotes both the group-divisible scheme with $n$ blocks of size $m$ and also $n$ copies of the trivial orthogonal block structure on an $m$-set.

We can now define the new way of combining two orthogonal block structures.

Definition. For $r=1,2$, let $\mathcal{F}_{r}$ be an orthogonal block structure on a set $\Omega_{r}$ and let $F_{r} \in \mathcal{F}_{r}$. The (simple) crested product of $\mathcal{F}_{1}$ and $\mathcal{F}_{2}$ with respect to $F_{1}$ and $F_{2}$ is the following set $\mathcal{G}$ of partitions of $\Omega_{1} \times \Omega_{2}$ :

$$
\mathcal{G}=\left\{G_{1} \times G_{2}: G_{1} \in \mathcal{F}_{1}, G_{2} \in \mathcal{F}_{2}, G_{1} \preccurlyeq F_{1} \text { or } G_{2} \succcurlyeq F_{2}\right\} .
$$

THEOREM 1. The crested product, as just defined, is an orthogonal block structure on $\Omega_{1} \times \Omega_{2}$.

Proof. All partitions in $\mathcal{G}$ are uniform, and all their relation matrices commute with each other. The two trivial partitions $U_{1} \times U_{2}$ and $E_{1} \times E_{2}$ of $\Omega_{1} \times \Omega_{2}$ are in $\mathcal{G}$, because $U_{2} \succcurlyeq F_{2}$ and $E_{1} \preccurlyeq F_{1}$. Suppose that $G_{1} \times G_{2}$ and $H_{1} \times H_{2}$ are both in $\mathcal{G}$. Then $\left(G_{1} \times G_{2}\right) \wedge\left(H_{1} \times H_{2}\right)=\left(G_{1} \wedge H_{1}\right) \times\left(G_{2} \wedge H_{2}\right)$ and $\left(G_{1} \times G_{2}\right) \vee\left(H_{1} \times H_{2}\right)=$ $\left(G_{1} \vee H_{1}\right) \times\left(G_{2} \vee H_{2}\right)$. If $G_{1} \preccurlyeq F_{1}$ or $H_{1} \preccurlyeq F_{1}$ then $G_{1} \wedge H_{1} \preccurlyeq F_{1}$ and so $\left(G_{1} \times G_{2}\right) \wedge\left(H_{1} \times H_{2}\right) \in \mathcal{G}$; otherwise, $G_{2} \succcurlyeq F_{2}$ and $H_{2} \succcurlyeq F_{2}$ so $G_{2} \wedge H_{2} \succcurlyeq F_{2}$ and so $\left(G_{1} \times G_{2}\right) \wedge\left(H_{1} \times H_{2}\right) \in \mathcal{G}$. Similarly, $\left(G_{1} \times G_{2}\right) \vee\left(H_{1} \times H_{2}\right) \in \mathcal{G}$.

If $F_{1}=U_{1}$ or $F_{2}=E_{2}$ then $\mathcal{G}$ is $\mathcal{F}_{1} \times \mathcal{F}_{2}$; if $F_{1}=E_{1}$ and $F_{2}=U_{2}$ then $\mathcal{G}=\mathcal{F}_{1} / \mathcal{F}_{2}$. Thus both crossing and nesting are special cases of the crested product. The word "crested" is a mixture of "crossed" and "nested" and is also cognate with the meaning of "wreath" in "wreath product".

EXAMPLE 1. Any Latin square of order $n$ defines an orthogonal block structure on the set of $n^{2}$ cells of the square: the nontrivial partitions $R, C$ and $L$ have as their 
parts the rows, columns and letters respectively. Take $\mathcal{F}_{1}$ to be such an orthogonal block structure and $\mathcal{F}_{2}$ to be trivial. The crested product of $\mathcal{F}_{1}$ and $\mathcal{F}_{2}$ with respect to $L$ and $U_{2}$ has the Hasse diagram shown in Figure 1.

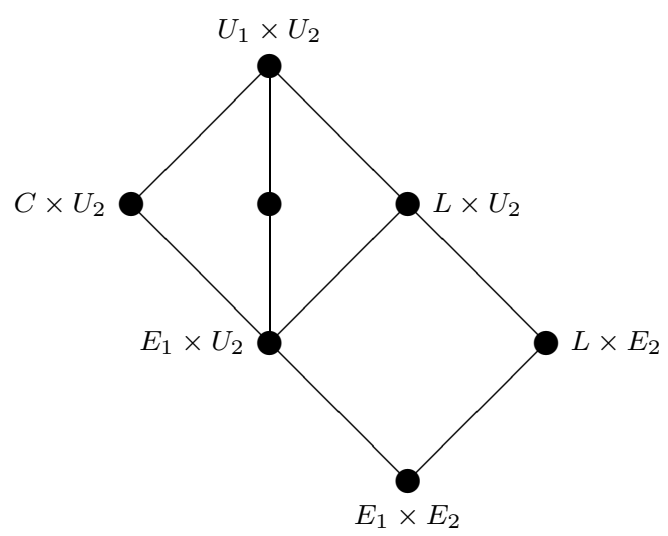

FIGURE 1. Crested product of a Latin square and a trivial orthogonal block structure

An important subclass of orthogonal block structures consists of the poset block structures. Suppose that $\leqslant$ is a partial order on a finite set $X$. A subset $Y$ of $X$ is defined to be ancestral if $y \in Y$ whenever $x \in Y$ and $x \leqslant y$. Denote by $\mathcal{S}(X)$ the set of ancestral subsets of $X$. For $x$ in $X$, let $\Gamma_{x}$ be a set of finite cardinality greater than 1 , and put $\Omega=\prod_{x \in X} \Gamma_{x}$. Each subset $Y$ of $X$ defines a partition $F(Y)$ of $\Omega$ as follows: $\left(\gamma_{x}: x \in X\right)$ and $\left(\delta_{x}: x \in X\right)$ are in the same part of $F(Y)$ if and only if $\gamma_{x}=\delta_{x}$ for all $x$ in $Y$. Note that $F(Y) \preccurlyeq F(Z)$ if and only if $Z \subseteq Y$. It is shown in [2] that $\{F(Y): Y \in \mathcal{S}(X)\}$ is an orthogonal block structure on $\Omega$. It is called a poset block structure.

THEOREM 2. Crested products of poset block structures are poset block structures.

Proof. For $r=1,2$, let $\leqslant_{r}$ be a partial order on $X_{r}$. Assume that $X_{1} \cap X_{2}=\emptyset$, and put $X=X_{1} \cup X_{2}$. Let $\Omega_{1}=\prod_{x \in X_{1}} \Gamma_{x}$ and $\Omega_{2}=\prod_{x \in X_{2}} \Gamma_{x}$, where each $\left|\Gamma_{x}\right|$ is finite and at least 2. Then $\Omega_{1} \times \Omega_{2}=\prod_{x \in X} \Gamma_{x}$. For $r=1,2$, let $\mathcal{P}_{r}$ be the poset block structure on $\Omega_{r}$ defined by $\left(X_{r}, \leqslant_{r}\right)$, and let $Y_{r}$ be an ancestral subset of $X_{r}$. If $\mathcal{P}$ is the crested product of $\mathcal{P}_{1}$ and $\mathcal{P}_{2}$ with respect to $F\left(Y_{1}\right)$ and $F\left(Y_{2}\right)$ then $\mathcal{P}=\{F(Z): Z \in \mathcal{T}\}$ where

$$
\mathcal{T}=\left\{Z_{1} \cup Z_{2}: Z_{1} \in \mathcal{S}\left(X_{1}\right), Z_{2} \in \mathcal{S}\left(X_{2}\right), Z_{1} \supseteq Y_{1} \text { or } Z_{2} \subseteq Y_{2}\right\} .
$$

The elements of $\mathcal{T}$ are precisely those subsets of $X$ which are ancestral for the partial order $\leqslant$ defined by

$$
x \leqslant y \quad \text { if } \quad\left\{\begin{array}{l}
x \in X_{1}, y \in X_{1} \text { and } x \leqslant 1 y, \text { or } \\
x \in X_{2}, y \in X_{2} \text { and } x \leqslant 2 y, \text { or } \\
x \in X_{2} \backslash Y_{2} \text { and } y \in Y_{1} .
\end{array}\right.
$$

Hence $\mathcal{P}$ is the poset block structure on $\Omega_{1} \times \Omega_{2}$ defined by $(X, \leqslant)$. 
If $Y_{1}=\emptyset$ or $Y_{2}=X_{2}$ then $\mathcal{P}=\mathcal{P}_{1} \times \mathcal{P}_{2}$ and $(X, \leqslant)$ is the cardinal sum of the posets $\left(X_{1}, \leqslant_{1}\right)$ and $\left(X_{2}, \leqslant_{2}\right)$; if $Y_{1}=X_{1}$ and $Y_{2}=\emptyset$ then $\mathcal{P}=\mathcal{P}_{1} / \mathcal{P}_{2}$ and $(X, \leqslant)$ is the ordinal sum of the posets $\left(X_{1}, \leqslant_{1}\right)$ and $\left(X_{2}, \leqslant_{2}\right)$. Thus Equation $(2.1)$ is a way of combining two disjoint posets that generalizes both cardinal sum and ordinal sum.

When only crossing and nesting are available as binary operators on orthogonal block structures, not all poset block structures can be built up from trivial ones. For example, the poset

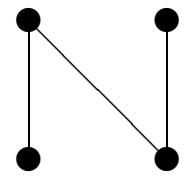

cannot be obtained from singleton posets by repeated use of cardinal and/or ordinal sum. Crested products change this completely.

THEOREM 3. Every poset block structure can be obtained from trivial poset block structures by repeated use of crested products.

Proof. It suffices to prove that every finite poset can be built from two disjoint smaller posets by means of Construction (2.1).

Let $(X, \leqslant)$ be a finite poset and let $y$ be a maximal element of $X$. Put $X_{1}=$ $Y_{1}=\{y\}, X_{2}=X \backslash\{y\}$ and $Y_{2}=X_{2} \backslash\left\{x \in X_{2}: x<y\right\}$. Let $\leqslant_{1}$ be the trivial partial order on $X_{1}$ and let $\leqslant 2$ be the restriction of $\leqslant$ to $X_{2}$. Then $Y_{1} \in \mathcal{S}\left(X_{1}\right)$, $Y_{2} \in \mathcal{S}\left(X_{2}\right)$ and $\leqslant$ is obtained from $\leqslant_{1}$ and $\leqslant_{2}$ by Construction $(2.1)$.

Theorem 3 does not extend to orthogonal block structures. The lattice of partitions in an orthogonal block structure is modular, so the lattice has a well-defined height. Since the crested product of $\mathcal{F}_{1}$ and $\mathcal{F}_{2}$ always contains

$$
F \times U_{2} \quad \text { for all } F \text { in } \mathcal{F}_{1}
$$

and

$$
E_{1} \times G \quad \text { for all } G \text { in } \mathcal{F}_{2},
$$

the height of the crested product is the sum of the heights of $\mathcal{F}_{1}$ and $\mathcal{F}_{2}$.

It follows that the only crested products of height two are $\underline{\underline{n}} / \underline{\underline{m}}$ and $\underline{\underline{n}} \times \underline{\underline{m}}$. The orthogonal block structure defined by a Latin square in Example 1 has height two and cannot be obtained as a crested product. Nor can the other orthogonal block structures of height two: these are obtained from sets of mutually orthogonal Latin squares, with $t+2$ non-trivial partitions if there are $t$ squares.

EXAMPLE 2. Similar arguments show that the orthogonal block structure shown in Figure 2 is not a crested product. Here $\Omega$ is the group

$$
\left\langle a, b: a^{9}=b^{3}=1, b^{-1} a b=a^{4}\right\rangle .
$$

Each subgroup of $\Omega$ defines a uniform partition of $\Omega$ into its left cosets. In this group all subgroups commute in pairs, so these partitions do form an orthogonal block structure, which has height three. Examination of all possible crested products of 
orthogonal block structures with heights one and two shows that none of them is the one in Figure 2.

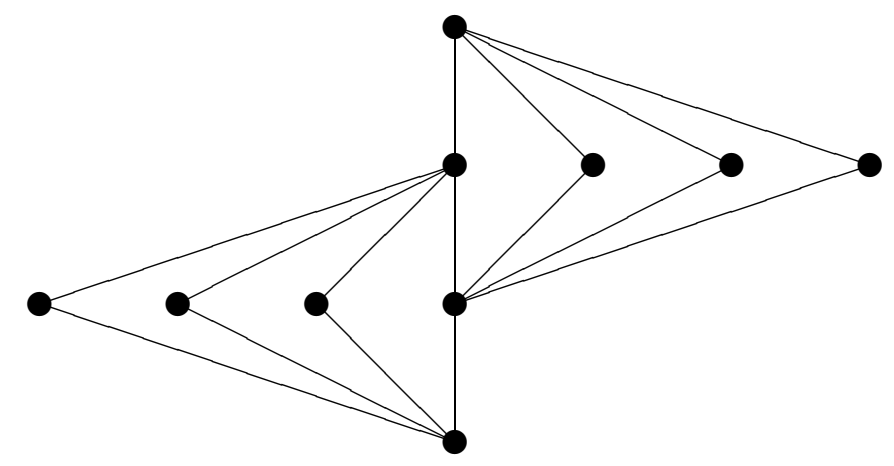

FIGURE 2. Orthogonal block structure defined by the subgroups of the group in Example 2

\section{Partitions in association schemes}

Definition. Let $\mathcal{Q}$ be an association scheme on $\Omega$ with adjacency matrices $A_{i}$ for $i$ in $\mathcal{K}$. A partition $F$ of $\Omega$ is inherent in $\mathcal{Q}$ if there is a subset $\mathcal{L}$ of $\mathcal{K}$ such that $R_{F}=\sum_{i \in \mathcal{L}} A_{i}$.

The trivial partitions $E$ and $U$ are inherent in every association scheme. If there are no other inherent partitions then the association scheme is called primitive; otherwise it is imprimitive: see [11]. Thus all non-trivial orthogonal block structures are imprimitive.

EXAmple 3. There is an association scheme on the 12 edges of the cube. Distinct edges $\alpha$ and $\beta$ are related by relation

$$
\begin{array}{ll}
1 & \text { if } \alpha \text { and } \beta \text { meet at a vertex } \\
2 & \text { if } \alpha \text { and } \beta \text { are diagonally opposite } \\
3 & \text { if } \alpha \text { and } \beta \text { are parallel but not opposite } \\
4 & \text { if } \alpha \text { and } \beta \text { are skew. }
\end{array}
$$

The partitions inherent in the association scheme have relation matrices $A_{0}, A_{0}+A_{2}$, $A_{0}+A_{2}+A_{3}$ and $A_{0}+A_{1}+A_{2}+A_{3}+A_{4}$.

THEOREM 4. If $\mathcal{Q}$ is an association scheme on $\Omega$ then the set $\mathcal{F}$ of partitions of $\Omega$ which are inherent in $\mathcal{Q}$ is an orthogonal block structure on $\Omega$.

Proof. Every adjacency matrix of $\mathcal{Q}$ has constant row-sums, so every partition in $\mathcal{F}$ is uniform. Moreover, $\mathcal{F}$ contains $U$ and $E$.

Suppose that $F$ and $G$ are in $\mathcal{F}$. Then $R_{F}$ and $R_{G}$ are in the Bose-Mesner 
algebra $\mathcal{A}$ of $\mathcal{Q}$, which is commutative, so $R_{F}$ commutes with $R_{G}$. If $R_{F}=\sum_{i \in \mathcal{L}} A_{i}$ and $R_{G}=\sum_{i \in \mathcal{M}} A_{i}$ then $R_{F \wedge G}=\sum_{i \in \mathcal{L} \cap \mathcal{M}} A_{i}$, and so $F \wedge G$ is in $\mathcal{F}$. In particular, $F \wedge G$ is uniform, and so Proposition 3 of [2] shows that $R_{F} R_{G}=k_{F \wedge G} R_{F \vee G}$ : therefore $R_{F \vee G} \in \mathcal{A}$ and so $F \vee G \in \mathcal{F}$.

Theorem 4 gives a more direct proof of Theorem 7 of $[\mathbf{3}]$.

EXAMPLE 4. Let $\Omega$ be an Abelian group and let $\mathcal{Q}$ be the association scheme on $\Omega$ in which $(\alpha, \beta)$ is in the same class as $(\gamma, \delta)$ if $\alpha^{-1} \beta \in\left\{\gamma^{-1} \delta, \delta^{-1} \gamma\right\}$. The partitions inherent in $\mathcal{Q}$ form the orthogonal block structure defined by all subgroups of $\Omega$ : see $[\mathbf{1}, \mathbf{9}]$.

Given any partition $\mathcal{P}$ of $\Omega \times \Omega$, let $V(\mathcal{P})$ be the span (over $\mathbb{R}$ ) of the adjacency matrices of its classes. Then $\mathcal{Q} \preccurlyeq \mathcal{P}$ if and only if $V(\mathcal{P}) \leqslant \mathcal{A}$.

Definition. Let $\mathcal{Q}$ be an association scheme on $\Omega$. A partition $\mathcal{P}$ of $\Omega \times \Omega$ is ideal for $\mathcal{Q}$ if $V(\mathcal{P})$ is an ideal of $\mathcal{A}$ in the sense that $V(\mathcal{P}) \leqslant \mathcal{A}$ and $A D \in V(\mathcal{P})$ whenever $A \in \mathcal{A}$ and $D \in V(\mathcal{P})$.

Inherent partitions were introduced in [11] in order to define quotient schemes. The calculations in the proof of Theorem 9.4 of $[\boldsymbol{7}]$ show that if $F$ is an inherent partition of $\mathcal{Q}$ then there is an ideal partition $\vartheta(F)$ for $\mathcal{Q}$ such that $A_{i} R_{F}$ is an integer multiple of an adjacency matrix of $\vartheta(F)$ for all $i$ in $\mathcal{K}$. The following result shows that $\vartheta$ is a bijection.

Theorem 5. Let $\mathcal{P}$ be an ideal partition for $\mathcal{Q}$. Let the adjacency matrices for $\mathcal{Q}$ be $A_{i}$ for $i$ in $\mathcal{K}$, and those for $\mathcal{P}$ be $D_{m}$ for $m$ in $\mathcal{M}$. Denote by $\sigma$ the surjection from $\mathcal{K}$ to $\mathcal{M}$ such that class $i$ of $\mathcal{Q}$ is contained in class $\sigma(i)$ of $\mathcal{P}$. Put $R=D_{\sigma(0)}$. Then $R$ is the relation matrix of an inherent partition in $\mathcal{Q}$. Moreover, for all $i$ in $\mathcal{K}, A_{i} R$ is an integer multiple of $D_{\sigma(i)}$.

Proof. First fix $i$ in $\mathcal{K}$. Because $\mathcal{P}$ is an ideal partition, there are integers $t_{m}$, for $m$ in $\mathcal{M}$, such that $A_{i} R=\sum_{m \in \mathcal{M}} t_{m} D_{m}$. If $m$ and $n$ are distinct elements of $\mathcal{M}$ then the diagonal elements of $D_{m} D_{n}$ are zero; while those of $D_{m}^{2}$ are equal to the constant row-sum $d_{m}$ of $D_{m}$. Therefore the diagonal elements of $A_{i} R D_{m}$ are equal to $t_{m} d_{m}$. If $m \neq \sigma(i)$ then the diagonal elements of $A_{i} D_{m}$ are zero, but $A_{i} D_{m} \in V(\mathcal{P})$ so there are integers $u_{l}$, for $l$ in $\mathcal{M} \backslash\{\sigma(0)\}$, such that

$$
A_{i} D_{m}=\sum_{l \in \mathcal{M} \backslash\{\sigma(0)\}} u_{l} D_{l} .
$$

Then $A_{i} R D_{m}=A_{i} D_{m} R=A_{i} D_{m} D_{\sigma(0)}=\sum_{l \neq \sigma(0)} u_{l} D_{l} D_{\sigma(0)}$, whose diagonal elements are zero, so $t_{m}=0$. Hence $A_{i} R=t_{\sigma(i)} D_{\sigma(i)}$.

Now put $\mathcal{L}=\{i \in \mathcal{K}: \sigma(i)=\sigma(0)\}$. Then $R=\sum_{i \in \mathcal{L}} A_{i}$ and $A_{i} R$ is an integer multiple of $R$ for all $i$ in $\mathcal{L}$. Consequently $R^{2}$ is an integer multiple of $R$ and so $R$ is the relation matrix of a uniform partition of $\Omega$. This partition is inherent because $R$ is in the Bose-Mesner algebra of $\mathcal{Q}$.

In the quotient association scheme of $\mathcal{Q}$ by its inherent partition $F$, the objects 
are the parts of $F$ and the adjacency matrices are the collapsed versions of the adjacency matrices of $\vartheta(F)$.

\section{Products of association schemes}

Let $F$ be a partition in an orthogonal block structure $\mathcal{F}$. Then $R_{F}=\sum_{G \in \mathcal{L}} A_{G}$, where $\mathcal{L}=\{G \in \mathcal{F}: G \preccurlyeq F\}$. Hence $F$ is inherent in the association scheme $\mathcal{Q}$ derived from $\mathcal{F}$. Let $\mathcal{A}$ be the Bose-Mesner algebra of $\mathcal{Q}$. Then $\left\{A_{G}: G \in \mathcal{L}\right\}$ and $\left\{R_{G}: G \in \mathcal{L}\right\}$ span the same subspace $\left.\mathcal{A}\right|_{F}$ of $\mathcal{A}$, and this is closed under matrix multiplication.

Let $\mathcal{P}$ be the ideal partition $\vartheta(F)$. For $G$ in $\mathcal{F}, R_{G}$ is in the ideal of $\mathcal{A}$ generated by $R_{F}$ if and only if $F \preccurlyeq G$ : therefore $V(\mathcal{P})$ is the span of $\left\{R_{G}: G \in \mathcal{F}, G \succcurlyeq F\right\}$. Henceforth write $\left.\mathcal{A}\right|^{F}$ for $V(\vartheta(F))$.

Now let $\mathcal{G}$ be the crested product of orthogonal block structures $\mathcal{F}_{1}$ and $\mathcal{F}_{2}$ with respect to $F_{1}$ and $F_{2}$. The span of the relation matrices of the partitions in $\mathcal{G}$ is

$$
\left(\left.\mathcal{A}_{1}\right|_{F_{1}} \otimes \mathcal{A}_{2}\right)+\left(\left.\mathcal{A}_{1} \otimes \mathcal{A}_{2}\right|^{F_{2}}\right)
$$

where $\mathcal{A}_{1}$ and $\mathcal{A}_{2}$ are the Bose-Mesner algebras of the association schemes defined by $\mathcal{F}_{1}$ and $\mathcal{F}_{2}$. The adjacency matrices of the association scheme defined by $\mathcal{G}$ are $(0,1)$-matrices which sum to $J_{\Omega_{1} \times \Omega_{2}}$ and which span this algebra. Therefore they are

$$
A_{G} \otimes A_{H} \quad \text { for } G \text { in } \mathcal{L} \text { and } H \text { in } \mathcal{F}_{2}
$$

and

$$
A_{G} \otimes D \quad \text { for } G \text { in } \mathcal{F}_{1} \backslash \mathcal{L} \text { and } D \text { an adjacency matrix of } \mathcal{P} \text {, }
$$

where $\mathcal{L}=\left\{G \in \mathcal{F}_{1}: G \preccurlyeq F_{1}\right\}$ and $\mathcal{P}=\vartheta\left(F_{2}\right)$. This motivates the following definition.

Definition. For $r=1,2$, let $\mathcal{Q}_{r}$ be an association scheme on a set $\Omega_{r}$, and let $F_{r}$ be an inherent partition in $\mathcal{Q}_{r}$. Put $\mathcal{P}=\vartheta\left(F_{2}\right)$ and $\Omega=\Omega_{1} \times \Omega_{2}$. Let the adjacency matrices of $\mathcal{Q}_{1}, \mathcal{Q}_{2}$ and $\mathcal{P}$ be $A_{i}$ for $i$ in $\mathcal{K}_{1}, B_{j}$ for $j$ in $\mathcal{K}_{2}$, and $D_{m}$ for $m$ in $\mathcal{M}$. Let $\mathcal{L}$ be the subset of $\mathcal{K}_{1}$ such that $R_{F_{1}}=\sum_{i \in \mathcal{L}} A_{i}$. The (simple) crested product of $\mathcal{Q}_{1}$ and $\mathcal{Q}_{2}$ with respect to $F_{1}$ and $F_{2}$ is the set $\mathcal{Q}$ of subsets of $\Omega \times \Omega$ whose adjacency matrices are

$$
A_{i} \otimes B_{j} \quad \text { for } i \text { in } \mathcal{L} \text { and } j \text { in } \mathcal{K}_{2}
$$

and

$$
A_{i} \otimes D_{m} \quad \text { for } i \text { in } \mathcal{K}_{1} \backslash \mathcal{L} \text { and } m \text { in } \mathcal{M} .
$$

THEOREM 6. The crested product, as just defined, is an association scheme on $\Omega$.

Proof. All of the matrices are symmetric. There are distinguished elements 0 in both $\mathcal{L}$ and $\mathcal{K}_{2}$ such that $A_{0}=I_{\Omega_{1}}$ and $B_{0}=I_{\Omega_{2}}$ : then $A_{0} \otimes B_{0}=I_{\Omega}$.

We have

$$
\sum_{i \in \mathcal{L}} \sum_{j \in \mathcal{K}_{2}}\left(A_{i} \otimes B_{j}\right)=\left(\sum_{i \in \mathcal{L}} A_{i}\right) \otimes\left(\sum_{j \in \mathcal{K}_{2}} B_{j}\right)=R_{F_{1}} \otimes J_{\Omega_{2}}
$$


and

$$
\sum_{i \in \mathcal{K}_{1} \backslash \mathcal{L}} \sum_{m \in \mathcal{M}}\left(A_{i} \otimes D_{m}\right)=\left(\sum_{i \in \mathcal{K}_{1} \backslash \mathcal{L}} A_{i}\right) \otimes\left(\sum_{m \in \mathcal{M}} D_{m}\right)=\left(J_{\Omega_{1}}-R_{F_{1}}\right) \otimes J_{\Omega_{2}}
$$

so the subsets of $\Omega \times \Omega$ defined by the adjacency matrices do form a partition of $\Omega \times \Omega$.

For $r=1,2$, let $\mathcal{A}_{r}$ be the Bose-Mesner algebra of $\mathcal{Q}_{r}$. Let $\left.\mathcal{A}_{1}\right|_{F_{1}}$ be the span of $\left\{A_{i}: i \in \mathcal{L}\right\}$. Then $\mathcal{A}_{1}, \mathcal{A}_{2},\left.\mathcal{A}_{1}\right|_{F_{1}}$ and $\left.\mathcal{A}_{2}\right|^{F_{2}}$ are all closed under multiplication. Define $\sigma: \mathcal{K}_{2} \rightarrow \mathcal{M}$ as in Theorem 5. Then

$$
\sum_{j: \sigma(j)=m} A_{i} \otimes B_{j}=A_{i} \otimes D_{m}
$$

and so the span $\mathcal{A}$ of the adjacency matrices is $\left(\left.\mathcal{A}_{1}\right|_{F_{1}} \otimes \mathcal{A}_{2}\right)+\left(\left.\mathcal{A}_{1} \otimes \mathcal{A}_{2}\right|^{F_{2}}\right)$. Each of $\left(\left.\mathcal{A}_{1}\right|_{F_{1}} \otimes \mathcal{A}_{2}\right)$ and $\left(\left.\mathcal{A}_{1} \otimes \mathcal{A}_{2}\right|^{F_{2}}\right)$ is closed under matrix multiplication. If $i_{1} \in \mathcal{L}$, $j \in \mathcal{K}_{2}, i_{2} \in \mathcal{K}_{1}$ and $m \in \mathcal{M}$ then $A_{i_{1}} A_{i_{2}} \in \mathcal{A}_{1}$ and $\left.B_{j} D_{m} \in \mathcal{A}_{2}\right|^{F_{2}}$, because $\mathcal{P}$ is ideal, and so $\left(A_{i_{1}} \otimes B_{j}\right)\left(A_{i_{2}} \otimes D_{m}\right) \in \mathcal{A}$. Hence $\mathcal{A}$ is closed under multiplication and so $\mathcal{Q}$ is an association scheme.

Again, the crested product reduces to the direct product if $F_{1}=U_{1}$ or $F_{2}=E_{2}$ (in which case $\mathcal{P}=\mathcal{Q}_{2}$ ); and it reduces to the wreath product if $F_{1}=E_{1}$ and $F_{2}=U_{2}$ (in which case $\mathcal{P}=U_{\Omega_{2} \times \Omega_{2}}$ ). The crested product is always imprimitive, because it contains the inherent partition $E_{1} \times U_{2}$. The subscheme induced on each class of $E_{1} \times U_{2}$ is isomorphic to $\mathcal{Q}_{2}$, while the quotient scheme is isomorphic to $\mathcal{Q}_{1}$. This gives a flexible method of constructing an 'extension' of one association scheme by another.

\section{The character table of a crested product}

Since the Bose-Mesner algebra $\mathcal{A}$ of an association scheme $\mathcal{Q}$ is commutative, it has common eigenspaces, called strata by statisticians. The stratum projectors $S_{e}$, for $e$ in $\mathcal{E}$, are also known as primitive idempotents. They form an alternative basis for $\mathcal{A}$, so $|\mathcal{K}|=|\mathcal{E}|$ : see [12, Chapter 17]. The character table of $\mathcal{Q}$ is the $\mathcal{K} \times \mathcal{E}$ matrix whose entry $C(i, e)$ is the eigenvalue of $A_{i}$ on stratum $W_{e}$ (this matrix is called $P$ in $[\mathbf{1 2}])$.

If $F$ is an inherent partition in $\mathcal{Q}$ then $k_{F}^{-1} R_{F}$ is idempotent so there is a subset $\mathcal{H}$ of $\mathcal{E}$ such that $R_{F}=k_{F} \sum_{e \in \mathcal{H}} S_{e}$. Define the equivalence relation $\sim$ on $\mathcal{E}$ by $e \sim f$ if and only if $C(i, e)=C(i, f)$ for all $i$ in $\mathcal{L}$, and write $[e]$ for the equivalence class containing $e$. Then $\mathcal{H}=[0]$. The classes of each of the subschemes defined by $F$ are indexed by $\mathcal{L}$ while the strata of each are indexed by the equivalence classes of $\sim$. The Bose-Mesner algebra of each subscheme is isomorphic to $\left.\mathcal{A}\right|_{F}$.

On the other hand, the classes of the quotient scheme are indexed by the classes of $\vartheta(F)$ while the stratum projectors are collapsed versions of $S_{e}$ for $e$ in $\mathcal{H}$. The linear map $\tau$ that takes $k_{F}^{-1} D_{m}$ to the $m$-th adjacency matrix of the quotient scheme is an isomorphism from $\left.\mathcal{A}\right|^{F}$ to the Bose-Mesner algebra of the quotient scheme.

TheOREM 7. Let $\mathcal{Q}$ be the crested product defined in Section 4. Let the strata for $\mathcal{Q}_{1}$ be $W_{e}$, for e in $\mathcal{E}_{1}$, with stratum projectors $S_{e}$, and those for $\mathcal{Q}_{2}$ be $V_{f}$, for $f$ in $\mathcal{E}_{2}$, with stratum projectors $T_{f}$. For $r=1,2$, let $C_{r}$ be the character table of $\mathcal{Q}_{r}$. 
In $\mathcal{Q}_{1}$, put $e \sim f$ if $C_{1}(i, e)=C_{1}(i, f)$ for all $i$ in $\mathcal{L}$, and put $U_{[e]}=\bigoplus_{f \sim e} W_{f}$, whose projector is equal to $\sum_{f \sim e} S_{f}$. In $\mathcal{Q}_{2}$, let $\mathcal{H}$ be the subset of $\mathcal{E}_{2}$ such that $R_{F_{2}}=k_{F_{2}} \sum_{f \in \mathcal{H}} T_{f}$. Then the strata for $\mathcal{Q}$ are $W_{e} \otimes V_{f}$, for $(e, f)$ in $\mathcal{E}_{1} \times \mathcal{H}$, and $U_{[e]} \otimes V_{f}$, for equivalence classes $[e]$ of $\sim$ and $f$ in $\mathcal{E}_{2} \backslash \mathcal{H}$. The eigenvalues are as follows, where $\bar{C}_{2}$ is the character table of the quotient $\overline{\mathcal{Q}}_{2}$ of $\mathcal{Q}_{2}$ by $F_{2}$.

\begin{tabular}{cc|c|c} 
& & $W_{e} \otimes V_{f} \quad(f \in \mathcal{H})$ & $U_{[e]} \otimes V_{f} \quad(f \notin \mathcal{H})$ \\
\hline$A_{i} \otimes D_{m}$ & $(i \notin \mathcal{L})$ & $C_{1}(i, e) k_{F_{2}} \bar{C}_{2}(m, f)$ & 0 \\
\hline$A_{i} \otimes B_{j}$ & $(i \in \mathcal{L})$ & $C_{1}(i, e) C_{2}(j, f)$ & $C_{1}(i, e) C_{2}(j, f)$
\end{tabular}

Proof. The claimed strata are mutually orthogonal and sum to $\mathbb{R}^{\Omega_{1} \times \Omega_{2}}$.

If $f \notin \mathcal{H}$ then $R_{F_{2}} T_{f}=0$ and so $D_{m} T_{f}=0$ because $D_{m}$ is a multiple of $R_{F_{2}}$. The other eigenvalues given follow directly from the character tables of the two schemes, the definition of $\sim$ and the isomorphism $\tau$. Thus to show that none of the claimed strata merge into a single stratum it is sufficient to show that we have the correct number of strata.

The number of equivalence classes of $\sim$ is equal to the rank of the subscheme of $\mathcal{Q}_{1}$ induced on each part of $F_{1}$, which is $|\mathcal{L}|$, while $|\mathcal{M}|$ and $|\mathcal{H}|$ are both equal to the rank of $\overline{\mathcal{Q}}_{2}$. Hence the number of adjacency matrices and the number of claimed strata are both equal to $\left|\mathcal{K}_{1}\right| \cdot|\mathcal{H}|-|\mathcal{L}| \cdot|\mathcal{H}|+|\mathcal{L}| \cdot\left|\mathcal{K}_{2}\right|$.

\section{Dual association schemes}

Association schemes $\mathcal{Q}$ and $\mathcal{Q}^{*}$ on sets of the same size $n$ are said to be formally dual [12, Chapter 17] if there is a bijection $*$ from the classes of $\mathcal{Q}$ to the strata of $\mathcal{Q}^{*}$ and from the strata of $\mathcal{Q}$ to the classes of $\mathcal{Q}^{*}$ such that

$$
C^{*}\left(e^{*}, i^{*}\right)=n C^{-1}(e, i)
$$

for all $(i, e)$ in $\mathcal{K} \times \mathcal{E}$, where $C$ and $C^{*}$ are the character tables of $\mathcal{Q}$ and $\mathcal{Q}^{*}$ respectively. Note that Equation (17.14) of [12] shows that

$$
C^{-1}(e, i)=C(i, e) r_{e} /\left(n v_{i}\right),
$$

where $r_{e}$ is the rank of $S_{e}$ and $v_{i}$ is the valency of $\mathcal{C}_{i}$.

Suppose that $\mathcal{Q}$ has such a dual, and that $F$ is an inherent partition in $\mathcal{Q}$ with $R_{F}=\sum_{i \in \mathcal{L}} A_{i}=k_{F} \sum_{e \in \mathcal{H}} S_{e}$. Then

$$
\sum_{i \in \mathcal{L}} A_{i}=k_{F} \sum_{e \in \mathcal{H}} \sum_{j \in \mathcal{K}} C^{-1}(e, j) A_{j}
$$

so $\sum_{e \in \mathcal{H}} C^{-1}(e, j)$ is equal to $1 / k_{F}$ if $j \in \mathcal{L}$ and to zero otherwise. Hence, in $\mathcal{Q}^{*}$,

$$
\sum_{e \in \mathcal{H}} A_{e^{*}}=\sum_{e \in \mathcal{H}} \sum_{i \in \mathcal{K}} C^{*}\left(e^{*}, i^{*}\right) S_{i^{*}}=\sum_{i \in \mathcal{K}} \sum_{e \in \mathcal{H}} n C^{-1}(e, i) S_{i^{*}}=\frac{n}{k_{F}} \sum_{i \in \mathcal{L}} S_{i^{*}} .
$$

Therefore $\sum_{e \in \mathcal{H}} A_{e^{*}}$ is the relation matrix of an inherent partition $F^{*}$ in $\mathcal{Q}^{*}$ whose parts have size $n / k_{F}$. We may call $F^{*}$ the inherent partition dual to $F$.

To proceed, we need to give more explicit results about the character table of a quotient scheme than we have used so far. The conclusion of Theorem 5 enables 
us to change the notation used in its proof so that $A_{i} R_{F}=t_{i} D_{\sigma(i)}$ for $i$ in $\mathcal{K}$. Moreover, $D_{\sigma(i)}=\sum_{j \in \sigma^{-1} \sigma(i)} A_{j}$. Then $v_{i} k_{F}=t_{i} d_{\sigma(i)}$ and $d_{\sigma(i)}=\sum_{j \in \sigma^{-1} \sigma(i)} v_{j}$.

Lemma 8. Let $\bar{C}$ be the character table of the quotient scheme $\overline{\mathcal{Q}}$ of $\mathcal{Q}$ by $F$. Then $\bar{C}(\sigma(i), e)=C(i, e) / t_{i}$ and $\bar{C}^{-1}(e, \sigma(i))=k_{F} C^{-1}(e, i)$ for e in $\mathcal{H}$ and $i$ in $\mathcal{K}$.

Proof. The primitive idempotents of $\overline{\mathcal{Q}}$ are $\tau\left(S_{e}\right)$ for $e$ in $\mathcal{H}$, and the adjacency matrices are the distinct $\tau\left(k_{F}^{-1} D_{\sigma(i)}\right)$. Now

$$
\begin{aligned}
\tau\left(k_{F}^{-1} D_{\sigma(i)}\right) & =\tau\left(t_{i}^{-1} A_{i} k_{F}^{-1} R_{F}\right) \\
& =\tau\left(t_{i}^{-1} A_{i}\right) \tau\left(k_{F}^{-1} R_{F}\right) \\
& =\tau\left(t_{i}^{-1} \sum_{e \in \mathcal{E}} C(i, e) S_{e}\right) \times I \\
& =\sum_{e \in \mathcal{E}} \frac{C(i, e)}{t_{i}} \tau\left(S_{e}\right)
\end{aligned}
$$

so $\bar{C}(\sigma(i), e)=C(i, e) / t_{i}$

The valency $v_{\sigma(i)}$ of $\tau\left(D_{\sigma(i)}\right)$ is just $v_{i} / t_{i}$, so Equation (6.2) shows that if there are $n$ points in the scheme $\mathcal{Q}$ then

$$
\bar{C}^{-1}(e, \sigma(i))=\frac{\bar{C}(\sigma(i), e) r_{e}}{\left(n / k_{F}\right) v_{\sigma(i)}}=\frac{C(i, e) k_{F} r_{e}}{t_{i} n\left(v_{i} / t_{i}\right)}=k_{F} C^{-1}(e, i) .
$$

The second part of this lemma shows that if $\sigma(i)=\sigma(j)$ then $C^{-1}(e, i)=$ $C^{-1}(e, j)$ for all $e$ in $\mathcal{H}$. Hence if Equation (6.1) holds then $C^{*}\left(e^{*}, i^{*}\right)=C^{*}\left(e^{*}, j^{*}\right)$ for all $e$ in $\mathcal{H}$, so $i^{*} \sim j^{*}$. Conversely, if $i^{*} \sim j^{*}$ and Equation (6.1) holds then $A_{i} \sum_{e \in \mathcal{H}} S_{e}$ is a scalar multiple of $A_{j} \sum_{e \in \mathcal{H}} S_{e}$, so $\sigma(i)=\sigma(j)$.

Incidentally, this lemma also shows that if $\mathcal{Q}$ is formally dual to $\mathcal{Q}^{*}$ and $F$ is inherent in $\mathcal{Q}$ then the quotient scheme of $\mathcal{Q}$ by $F$ is formally dual to the subschemes of $\mathcal{Q}^{*}$ induced on each part of $F^{*}$.

THEOREM 9. For $r=1,2$, suppose that $F_{r}$ is an inherent partition in the association scheme $\mathcal{Q}_{r}$ on a set of size $n_{r}$, and that $\mathcal{Q}_{r}^{*}$ is formally dual to $\mathcal{Q}_{r}$. Then the crested product $\mathcal{Q}^{*}$ of $\mathcal{Q}_{1}^{*}$ and $\mathcal{Q}_{2}^{*}$ with respect to $F_{1}^{*}$ and $F_{2}^{*}$ is formally dual to the crested product $\mathcal{Q}$ of $\mathcal{Q}_{2}$ and $\mathcal{Q}_{1}$ with respect to $F_{2}$ and $F_{1}$.

Proof. The adjacency matrices of $\mathcal{Q}$ are $A_{i} \otimes D_{m}$, for $i$ in $\mathcal{K}_{1} \backslash \mathcal{L}$ and $m$ in $\sigma\left(\mathcal{K}_{2}\right)$, and $A_{i} \otimes B_{j}$, for $i$ in $\mathcal{L}$ and $j$ in $\mathcal{K}_{2}$, while the strata are $W_{e} \otimes V_{f}$ for $e$ in $\mathcal{E}_{1}$ and $f$ in $\mathcal{H}$, and $U_{[e]} \otimes V_{f}$ for equivalence classes of $\sim$ on $\mathcal{E}_{1}$ and $f$ in $\mathcal{E}_{2} \backslash \mathcal{H}$. The adjacency matrices of $\mathcal{Q}^{*}$ are labelled by $\left(f^{*}, \sigma\left(e^{*}\right)\right)$ for $f$ in $\mathcal{E}_{2} \backslash \mathcal{H}$ and $e$ in $\mathcal{E}_{1}$, and by $\left(f^{*}, e^{*}\right)$ for $f$ in $\mathcal{H}$ and $e$ in $\mathcal{E}_{1}$, while its strata are labelled by $\left(j^{*}, i^{*}\right)$ for $j$ in $\mathcal{K}_{2}$ and $i$ in $\mathcal{L}$, and by $\left(\left[j^{*}\right], i^{*}\right)$ for $j$ in $\mathcal{K}_{2}$ and $i$ in $\mathcal{K}_{1} \backslash \mathcal{L}_{1}$.

Let $C$ and $C^{*}$ be the character tables of $\mathcal{Q}$ and $\mathcal{Q}^{*}$. We use Theorem 7 to obtain $C$, then Equation (6.2) to obtain $C^{-1}$, and show that it satisfies Equation (6.1) by using Theorem 7 for $\mathcal{Q}^{*}$.

First, take $i$ in $\mathcal{L}, j$ in $\mathcal{K}_{2}, e$ in $\mathcal{E}_{1}$ and $f$ in $\mathcal{H}$. Then $C((i, j),(e, f))=C_{1}(i, e) C_{2}(j, f)$. The valency of $A_{j} \otimes B_{j}$ is $v_{i} v_{j}$, so

$$
C^{-1}((e, f),(i, j))=\frac{C_{1}(i, e) C_{2}(j, f) r_{e} r_{f}}{n_{1} n_{2} v_{i} v_{j}}
$$




$$
\begin{aligned}
& =C_{1}^{-1}(e, i) C_{2}^{-1}(f, j) \\
& =\frac{C_{1}^{*}\left(e^{*}, i^{*}\right) C_{2}^{*}\left(f^{*}, j^{*}\right)}{n_{1} n_{2}} \\
& =\left(n_{1} n_{2}\right)^{-1} C^{*}\left(\left(f^{*}, e^{*}\right),\left(j^{*}, i^{*}\right) .\right.
\end{aligned}
$$

Secondly, take $i$ in $\mathcal{K}_{1} \backslash \mathcal{L}, j$ in $\mathcal{K}_{2}, e$ in $\mathcal{E}_{1}$ and $f$ in $\mathcal{H}$. Then

$$
C((i, \sigma(j)),(e, f))=C_{1}(i, e) k_{F_{2}} \bar{C}_{2}(\sigma(j), f)=C_{1}(i, e) k_{F_{2}} C_{2}(j, f) / t_{j} .
$$

The valency of $A_{i} \otimes D_{\sigma(j)}$ is $v_{i} v_{j} k_{F_{2}} / t_{j}$, so

$$
\begin{aligned}
C^{-1}((e, f),(i, \sigma(j)) & =\frac{C_{1}(i, e) k_{F_{2}} C_{2}(j, f) r_{e} r_{f}}{n_{1} n_{2} v_{i} v_{j} k_{F_{2}}} \\
& =C_{1}^{-1}(i, e) C_{2}^{-1}(j, f) \\
& =\frac{C_{1}^{*}\left(e^{*}, i^{*}\right) C_{2}^{*}\left(f^{*}, j^{*}\right)}{n_{1} n_{2}} \\
& =\left(n_{1} n_{2}\right)^{-1} C^{*}\left(\left(f^{*}, e^{*}\right),\left(j^{*}, i^{*}\right)\right) .
\end{aligned}
$$

Thirdly, take $i$ in $\mathcal{L}, j$ in $\mathcal{K}_{2}, e$ in $\mathcal{E}_{1}$ and $f$ in $\mathcal{E}_{2} \backslash \mathcal{H}$. Then $C((i, j),([e], f))=$ $C_{1}(i, e) C_{2}(j, f)$. Now the dimension of the stratum is $r_{f} \sum_{g \in[e]} r_{g}$. However,

$$
\sum_{g \in[e]} r_{g}=\sum_{g \in[e]} v_{g^{*}}=\sum_{g^{*} \in \sigma^{-1} \sigma(e)} v_{g^{*}}=k_{F_{1}^{*}} v_{e^{*}} / t_{e^{*}}=k_{F_{1}^{*}} r_{e} / t_{e^{*}},
$$

so

$$
\begin{aligned}
C^{-1}(([e], f),(i, j)) & =\frac{C_{1}(i, e) C_{2}(j, f) k_{F_{1}^{*}} r_{e} r_{f}}{n_{1} n_{2} v_{i} v_{j} t_{e^{*}}} \\
& =\frac{C_{1}^{-1}(e, i) C_{2}^{-1}(f, j) k_{F_{1}^{*}}}{t_{e^{*}}} \\
& =\frac{C_{1}^{*}\left(e^{*}, i^{*}\right) k_{F_{1}^{*}} C_{2}^{*}\left(f^{*}, j^{*}\right)}{n_{1} n_{2} t_{e^{*}}} \\
& =\frac{C_{2}^{*}\left(f^{*}, j^{*}\right) k_{F_{1}^{*}} \bar{C}_{1}^{*}\left(e^{*}, i^{*}\right)}{n_{1} n_{2}} \\
& =\left(n_{1} n_{2}\right)^{-1} C^{*}\left(\left(f^{*}, \sigma\left(e^{*}\right)\right),\left(j^{*}, i^{*}\right)\right) .
\end{aligned}
$$

Finally, take $i$ in $\mathcal{K}_{1} \backslash \mathcal{L}, j$ in $\mathcal{K}_{2}, e$ in $\mathcal{E}_{1}$ and $f$ in $\mathcal{E}_{2} \backslash \mathcal{H}$. Then $C((i, \sigma(i)),([e], f))=$ 0 and so $C^{-1}(([e], f),(i, \sigma(j)))=0$. However, $[e]=\sigma\left(e^{*}\right)$ and $\left[j^{*}\right]=\sigma(j)$, and $C^{*}\left(\left(f^{*}, \sigma\left(e^{*}\right),\left(\left[j^{*}\right], i^{*}\right)\right)=0\right.$.

\section{Products of permutation groups}

In this section we define the crested product of two permutation groups. We write permutations on the right, in contrast to other functions. We also warn of another source of confusion. We use $G^{\Omega}$ to denote the set of functions from $\Omega$ to $G$, not (as often in permutation group theory) the permutation group induced on the set $\Omega$ by the group $G$.

For $r=1,2$, let $G_{r}$ be a transitive permutation group on $\Omega_{r}$. We recall the definition of the wreath product $G_{2} \succ G_{1}$, which is a permutation group on $\Omega_{1} \times \Omega_{2}$ generated by the following two subgroups: 
(a) the base group $B=G_{2}^{\Omega_{1}}$, acting by the rule

$$
\left(\alpha_{1}, \alpha_{2}\right) \phi=\left(\alpha_{1}, \alpha_{2} \phi\left(\alpha_{1}\right)\right)
$$

for $\phi \in G_{2}^{\Omega_{1}}$;

(b) the top group $T=G_{1}$, acting by the rule

$$
\left(\alpha_{1}, \alpha_{2}\right) g=\left(\alpha_{1} g, \alpha_{2}\right)
$$

for $g \in G_{1}$.

Note that $T$ normalizes $B$, so the two groups generate their product; and $B \cap T=1$, so the product is semi-direct.

If we replace the base group $B$ by its subgroup $G_{2}$, embedded diagonally (that is, as the group of constant functions), we obtain the direct product $G_{1} \times G_{2}$.

Now suppose that, for $r=1,2$, we have a partition $F_{r}$ of $\Omega_{r}$ which is invariant under $G_{r}$. We need to make a further assumption: suppose that there is a normal subgroup $N$ of $G_{2}$ such that $F_{2}$ is the orbit partition of $N$. Now we may as well assume that $N$ consists of all elements of $G_{2}$ which fix all parts of the partition $F_{2}$. We use $F_{r}$ also to denote the set of parts of the partition $F_{r}$.

The base group $B$ of the simple crested product is the group generated by $N^{F_{1}}$ and $G_{2}$. Here $N^{F_{1}}$ is embedded in $G_{2}^{\Omega_{1}}$ as the set of functions which are constant on the classes of $F_{1}$ and take values in $N$; and $G_{2}$ is embedded diagonally, as before. Clearly $G_{2}$ normalizes $N^{F_{1}}$, so their product is a group; and the intersection is $N$ (embedded diagonally). The top group is $G_{1}$, which normalizes $B$; so the crested product is again a semi-direct product of $B$ by $G_{1}$. Its order is $|N|^{\left|F_{1}\right|-1} \cdot\left|G_{1}\right| \cdot\left|G_{2}\right|$. Clearly it contains the direct product and is contained in the wreath product of $G_{2}$ and $G_{1}$. Moreover, as usual, we see that it is equal to the direct product if $F_{2}=E_{2}$ or if $F_{1}=U_{1}$, and is equal to the wreath product if $F_{2}=U_{2}$ and $F_{1}=E_{1}$.

There are two concepts of an automorphism of an association scheme. In this paper we shall use the strong sense: a permutation of the underlying set that preserves all the classes of the scheme.

THEOREM 10. With the notation as above, suppose that $\mathcal{Q}_{r}$ is an association scheme on $\Omega_{r}$ admitting the group $G_{r}$, and that $F_{r}$ is an inherent partition in $\mathcal{Q}_{r}$, for $r=1,2$. Then the crested product of the two groups preserves the crested product of the two association schemes (relative to the partitions $F_{1}$ and $F_{2}$ in each case).

Proof. Since $\mathcal{Q}_{1} \times \mathcal{Q}_{2}$ refines the crested product and is preserved by $G_{1} \times G_{2}$, we only have to show that the group $N^{F_{1}}$ preserves the crested product. To show this we consider separately the two types of relation which might hold between two pairs $\left(\alpha_{1}, \alpha_{2}\right)$ and $\left(\beta_{1}, \beta_{2}\right)$, and apply an element $\phi$ in $N^{F_{1}}$ to both pairs.

If the matrix of the relation is $A_{i} \otimes B_{j}$ where $i \in \mathcal{L}$, then $F_{1}\left(\alpha_{1}\right)=F_{1}\left(\beta_{1}\right)$, and so the same element of $N$ is applied to $\alpha_{2}$ and $\beta_{2}$, so the $B_{j}$-relation they satisfy is preserved.

If the matrix is of the form $A_{i} \otimes D_{m}$, then the permutations $\phi\left(\alpha_{1}\right)$ and $\phi\left(\beta_{1}\right)$ do not change the $F_{2}$-parts of $\alpha_{2}$ and $\beta_{2}$ respectively; but the $D_{m}$-relation depends only on these $F_{2}$-parts.

We could have achieved Theorem 10 by being parsimonious about which permutations are included in the crested product of $G_{1}$ and $G_{2}$. The next result suggests that our definition is precisely the right one. 
THEOREM 11. Suppose that, for $r=1,2$, the classes of $\mathcal{Q}_{r}$ are the orbits of $G_{r}$ on $\Omega_{r} \times \Omega_{r}$. Let $F_{1}$ be an inherent partition in $\mathcal{Q}_{1}$ and $N$ a normal subgroup of $G_{2}$. Let $F_{2}$ be the orbit partition of $N$ on $\Omega_{2}$. Then $F_{2}$ is inherent in $\mathcal{Q}_{2}$, and the classes of the crested product $\mathcal{Q}$ of $\mathcal{Q}_{1}$ and $\mathcal{Q}_{2}$ with respect to $F_{1}$ and $F_{2}$ are the orbits of the crested product $G$ of $G_{1}$ and $G_{2}$ on $\left(\Omega_{1} \times \Omega_{2}\right) \times\left(\Omega_{1} \times \Omega_{2}\right)$.

Proof. Since $G_{2}$ centralizes $R_{F_{2}}$, the corresponding relation is a union of classes in $\mathcal{Q}_{2}$, so $F_{2}$ is inherent in $\mathcal{Q}_{2}$. Thus the crested product of the association schemes is defined. Even though $N$ need not be the whole partwise stabilizer of $F_{2}$, the crested product of the groups is defined. Now Theorem 10 shows that each class of $\mathcal{Q}$ is a union of orbits of $G$, so we have to show that $G$ is transitive on each class.

Suppose that $\left(\left(\alpha_{1}, \alpha_{2}\right),\left(\beta_{1}, \beta_{2}\right)\right)$ and $\left(\left(\gamma_{1}, \gamma_{2}\right),\left(\delta_{1}, \delta_{2}\right)\right)$ are in the same class of $\mathcal{Q}$. Then $\left(\alpha_{1}, \beta_{1}\right)$ and $\left(\gamma_{1}, \delta_{1}\right)$ are in the same class of $\mathcal{Q}_{1}$, so there is an element of the top group $G_{1}$ taking $\alpha_{1}$ to $\gamma_{1}$ and $\beta_{1}$ to $\delta_{1}$. Hence we may assume that $\alpha_{1}=\gamma_{1}$ and $\beta_{1}=\delta_{1}$.

If the class of $\mathcal{Q}$ has relation matrix $A_{i} \otimes B_{j}$ then $\left(\alpha_{2}, \beta_{2}\right)$ and $\left(\gamma_{2}, \delta_{2}\right)$ are in the same class of $\mathcal{Q}_{2}$. The crested product contains $G_{2}$ acting diagonally, and there is an element of $G_{2}$ taking $\left(\alpha_{2}, \beta_{2}\right)$ to $\left(\gamma_{2}, \delta_{2}\right)$.

Otherwise, the class of $\mathcal{Q}$ has relation matrix $A_{i} \otimes D_{m}$ for $i$ not in $\mathcal{L}$, so $\alpha_{1}$ and $\beta_{1}$ are not in the same part of $F_{1}$. There is some $j$ in $\mathcal{K}_{2}$ for which $D_{m}$ is a scalar multiple of $B_{j} R_{F_{2}}$. Hence there are points $\varepsilon$ and $\zeta$ in $\Omega_{2}$ such that $\left(\alpha_{2}, \varepsilon\right)$ and $\left(\gamma_{2}, \zeta\right)$ are in the $B_{j}$-relation while $\left(\varepsilon, \beta_{2}\right)$ and $\left(\zeta, \delta_{2}\right)$ are pairs in the same parts of $F_{2}$. There is an element $\phi_{0}$ in $G_{2}$ taking $\left(\alpha_{2}, \varepsilon\right)$ to $\left(\gamma_{2}, \zeta\right)$, and elements $h_{1}$ and $h_{2}$ in $N$ with $\varepsilon h_{1}=\beta_{2}$ and $\zeta h_{2}=\delta_{2}$. Since $\alpha_{1}$ and $\beta_{1}$ are in different parts of $F_{1}$, there are elements $\phi_{1}$ and $\phi_{2}$ of $N^{F_{1}}$ with $\phi_{1}\left(\alpha_{1}\right)=\phi_{2}\left(\alpha_{1}\right)=1, \phi_{1}\left(\beta_{1}\right)=$ $h_{1}^{-1}$ and $\phi_{2}\left(\beta_{1}\right)=h_{2}$. Now $G$ contains $\phi_{1} \phi_{0} \phi_{2}$ and $\left(\left(\alpha_{1}, \alpha_{2}\right),\left(\beta_{1}, \beta_{2}\right)\right) \phi_{1} \phi_{0} \phi_{2}=$ $\left(\left(\alpha_{1}, \alpha_{2}\right),\left(\beta_{1}, \varepsilon\right)\right) \phi_{0} \phi_{2}=\left(\left(\alpha_{1}, \gamma_{2}\right),\left(\beta_{1}, \zeta\right)\right) \phi_{2}=\left(\left(\alpha_{1}, \gamma_{2}\right),\left(\beta_{1}, \delta_{2}\right)\right)$.

REMARK. In fact, we can prove something a little stronger. A homogeneous coherent configuration [14] is a generalization of association scheme in which the symmetry condition is weakened to the condition that the transpose of every adjacency matrix is also an adjacency matrix. The crested product of homogeneous coherent configurations can be defined in just the same way as for association schemes. If $G_{r}$ is transitive on $\Omega_{r}$ then the orbits of $G_{r}$ on $\Omega_{r} \times \Omega_{r}$ form a homogeneous coherent configuration. The proofs of Theorems 10 and 11 show that the orbit partition of the crested product of two transitive groups is the crested product of the orbit partitions of the two groups.

REMARK. If the groups $G_{1}$ and $G_{2}$ have regular abelian subgroups $A_{1}$ and $A_{2}$, then the condition (implicit in Theorem 10) that the inherent partition $F_{2}$ is the orbit partition of a normal subgroup of $G_{2}$ is automatically satisfied. Also, the crested product has a regular abelian subgroup $A_{1} \times A_{2}$. In particular, the association scheme has a dual [13]. Theorem 9 shows that this is the crested product in reverse order of the duals of the two schemes (using the dual inherent partitions).

REMARK. There is another similar permutation group, obtained by taking instead the base group to be the product of $N^{\Omega_{1}}$ and $G_{2}^{F_{1}}$. Their intersection is $N^{F_{1}}$, and again the top group normalizes the base group. The new group is equal to 
the direct product if $F_{2}=E_{2}$ and $F_{1}=U_{1}$, and is equal to the wreath product if $F_{2}=U_{2}$ or if $F_{1}=E_{1}$. This suggests that there might be a different sort of crested product of association schemes, so that the analogue of the preceding theorem holds. In Section 8 we define a more general product which has both of these as special cases.

\section{A more general product of association schemes}

Let $\mathcal{F}$ be a set of inherent partitions of $\mathcal{Q}_{1}$ which contains $U_{1}$ and is closed under $\wedge$ and $\vee$ (except that we do not require that $\mathcal{F}$ contain the empty supremum $E$ ). For each $F$ in $\mathcal{F}$, let $\mathcal{L}_{F}$ be the set of indices in $\mathcal{K}_{1}$ such that $A_{i}$ is a component relation of $R_{F}$. Use Möbius inversion to obtain $\mathcal{J}_{F}$ as the set of $i$ such that $F$ is minimal subject to $i \in \mathcal{L}_{F}$. The sets $\mathcal{J}_{F}$, for $F$ in $\mathcal{F}$, partition $\mathcal{K}_{1}$, because $\mathcal{F}$ contains $U_{1}$ and is closed under $\wedge$. Write $F_{0}$ for the unique minimal partition in $\mathcal{F}$.

Further, suppose that $\psi$ is a map from $\mathcal{F}$ to the set of inherent partitions of $\mathcal{Q}_{2}$ with the properties that $\psi\left(F_{0}\right)=E_{2}$ and $\psi$ preserves order and suprema. Note that $\psi$ need not be one-to-one nor preserve infima. For $F$ in $\mathcal{F}$, write $\mathcal{D}_{F}$ for the set of adjacency matrices of the ideal partition $\vartheta(\psi(F))$.

The adjacency matrices of the extended crested product $\mathcal{Q}$ of $\mathcal{Q}_{1}$ and $\mathcal{Q}_{2}$ with respect to $\mathcal{F}$ and $\psi$ are

\section{$A_{i} \otimes D \quad$ for $i$ in $\mathcal{J}_{F}$ and $D$ in $\mathcal{D}_{F}$}

for all $F$ in $\mathcal{F}$. Clearly they are all symmetric. Since $\sum_{D \in \mathcal{D}_{F}} D=J_{\Omega_{2}}$, their sum is $\sum_{i \in \mathcal{K}_{1}} A_{i} \otimes J_{\Omega_{2}}$, which is $J_{\Omega_{1} \times \Omega_{2}}$. We have $I_{\Omega_{1}}=A_{0} \in \mathcal{J}_{F_{0}}$ and $I_{\Omega_{2}}=B_{0} \in \mathcal{D}_{F_{0}}=$ $\left\{B_{j}: j \in \mathcal{K}_{2}\right\}$ because $\psi\left(F_{0}\right)=E_{2}$, so $\mathcal{Q}$ contains $I_{\Omega_{1} \times \Omega_{2}}$. If $G \preccurlyeq F$ then $\psi(G) \preccurlyeq$ $\psi(F)$ so $\left.\mathcal{A}_{2}\right|^{\psi(F)} \leqslant\left.\mathcal{A}_{2}\right|^{\psi(G)}$ and therefore the span $\mathcal{A}$ of the adjacency matrices is $\sum_{F \in \mathcal{F}}\left[\left.\left.\mathcal{A}_{1}\right|_{F} \otimes \mathcal{A}_{2}\right|^{\psi(F)}\right]$. Consider partitions $F$ and $G$ in $\mathcal{F}$. The subalgebras of the subschemes are ordered in the same way as the inherent partitions, so $\left.\left.\mathcal{A}_{1}\right|_{F} \mathcal{A}_{1}\right|_{G} \subseteq$ $\left.\mathcal{A}_{1}\right|_{F \vee G}$. Also $\left.\left.\mathcal{A}_{2}\right|^{\psi(F)} \mathcal{A}_{2}\right|^{\psi(G)}$ is the ideal of $\mathcal{A}_{2}$ generated by $R_{\psi(F)} R_{\psi(G)}$, which is proportional to $R_{\psi(F) \vee \psi(G)}$, which is $R_{\psi(F \vee G)}$, so $\left.\left.\mathcal{A}_{2}\right|^{\psi(F)} \mathcal{A}_{2}\right|^{\psi(G)}=\left.\mathcal{A}_{2}\right|^{\psi(F \vee G)}$. Hence $\mathcal{A}$ is closed under multiplication and so $\mathcal{Q}$ is an association scheme.

Here are some special cases of the extended crested product.

(1) $\mathcal{F}=\left\{U_{1}\right\}, \psi\left(U_{1}\right)=E_{2}$ gives the direct product.

(2) $\mathcal{F}=\left\{E_{1}, U_{1}\right\}, \psi\left(X_{1}\right)=X_{2}$ for $X \in\{E, U\}$ gives the wreath product.

(3) $\mathcal{F}=\left\{F_{1}, U_{1}\right\}, \psi\left(F_{1}\right)=E_{2}, \psi\left(U_{1}\right)=F_{2}$ gives the simple crested product.

(4) $\mathcal{F}=\left\{E_{1}, F_{1}, U_{1}\right\}, \psi\left(X_{1}\right)=X_{2}$ for $X \in\{E, F, U\}$ gives the product hinted at in the final remark in Section 7.

It is curious that the 'non-standard' crested product has a neater definition in these terms than the 'standard' one does.

This more general version of the crested product still has $E_{1} \times U_{2}$ as an inherent partition whose subschemes are isomorphic to $\mathcal{Q}_{2}$ and whose quotient scheme is isomorphic to $\mathcal{Q}_{1}$. Nonetheless, there are, in general, many extensions of $\mathcal{Q}_{2}$ by $\mathcal{Q}_{1}$ that do not arise as crested products. Any Latin square of order $n$ is an extension of $\underline{\underline{n}}$ by $\underline{\underline{n}}$ but is not a crested product. See [18] for many more examples.

If $\mathcal{Q}_{r}$ is the association scheme of an orthogonal block structure $\mathcal{G}_{r}$ for $r=1,2$ then the extended crested product of $\mathcal{Q}_{1}$ and $\mathcal{Q}_{2}$ with respect to $\mathcal{F}$ and $\psi$ is the 
association scheme of the orthogonal block structure

$$
\bigcup_{F \in \mathcal{F}}\left\{H_{1} \times H_{2}: H_{1} \in \mathcal{G}_{1}, H_{2} \in \mathcal{G}_{2}, H_{1} \preccurlyeq F, H_{2} \succcurlyeq \psi(F)\right\} .
$$

It is not immediately obvious that this is closed under $\wedge$. However, suppose that $H_{1} \preccurlyeq F \in \mathcal{F}, K_{1} \preccurlyeq G \in \mathcal{F}, \psi(F) \preccurlyeq H_{2}$ and $\psi(G) \preccurlyeq K_{2}$. Then $H_{1} \wedge K_{1} \preccurlyeq F \wedge G \in$ $\mathcal{F}$, and $\psi(F \wedge G) \preccurlyeq \psi(F) \wedge \psi(G) \preccurlyeq H_{2} \wedge K_{2}$, because $\psi$ preserves order: hence $\left(H_{1} \wedge K_{1}\right) \times\left(H_{2} \wedge K_{2}\right)$ is in the orthogonal block structure.

EXAMPLE 5. Take four association schemes $\mathcal{Q}_{r s}$ for $r, s$ in $\{1,2\}$. For $r=1,2$, the direct product $\mathcal{Q}_{r 1} \times \mathcal{Q}_{r 2}$ has inherent partitions $E_{r 1} \times E_{r 2}, E_{r 1} \times U_{r 2}, U_{r 1} \times E_{r 2}$ and $U_{r 1} \times U_{r 2}$. Take $\psi\left(X_{1 s} \times Y_{1 s}\right)=X_{2 s} \times Y_{2 s}$ for $s$ in $\{1,2\}$ and $X, Y$ in $\{E, U\}$. Then the extended crested product of $\mathcal{Q}_{11} \times \mathcal{Q}_{12}$ and $\mathcal{Q}_{21} \times \mathcal{Q}_{22}$ with respect to $\psi$ is $\left(\mathcal{Q}_{11} / \mathcal{Q}_{21}\right) \times\left(\mathcal{Q}_{12} / \mathcal{Q}_{22}\right)$, so this construction does seem to precisely capture the idea of putting $F$ above $\psi(F)$ but no more.

EXAMPLE 6 . For $r=1,2$, let $\mathcal{Q}_{r}$ be the association scheme of the Latin square orthogonal block structure whose non-trivial partitions are $R_{r}, C_{r}$ and $L_{r}$. Put $\mathcal{F}=\left\{E_{1}, L_{1}, U_{1}\right\}$ and $\psi\left(X_{1}\right)=X_{2}$ for $X$ in $\{E, L, U\}$. The extended crested product is shown in Figure 3.

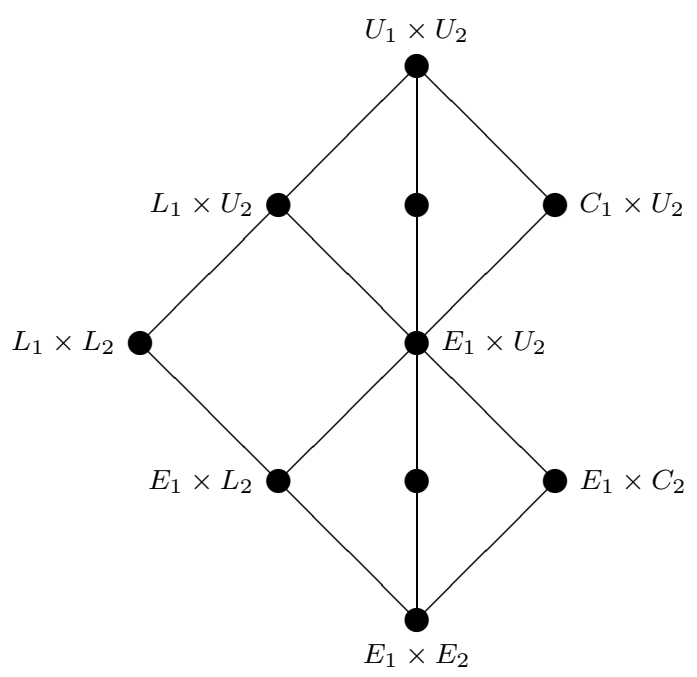

FiguRE 3. Extended crested product of two Latin squares

As before, the height of the lattice of the extended crested product of two orthogonal block structures is the sum of the original two heights. Examination of the small number of possible cases shows that the orthogonal block structure in Figure 3 cannot be attained from smaller structures by the simple crested product. Hence the extension gives something genuinely new. 


\section{A more general product of permutation groups}

We can make a similar definition of the extended crested product of two permutation groups $\left(G_{1}, \Omega_{1}\right)$ and $\left(G_{2}, \Omega_{2}\right)$.

Let $\mathcal{F}$ be a set of $G_{1}$-invariant partitions of $\Omega_{1}$.

Let $\mathcal{N}$ be a set of normal subgroups of $G_{2}$, closed under intersection (in particular, $\mathcal{N}$ contains the empty intersection $G_{2}$ ), and let $\rho$ be an order-preserving map from $\mathcal{F}$ onto $\mathcal{N}$. For $N$ in $\mathcal{N}$, let $F(N)$ be the orbit partition of $N$. If $N_{1}$ and $N_{2}$ are in $\mathcal{N}$ then $F\left(N_{1}\right)$ is orthogonal to $F\left(N_{2}\right)$ and $F\left(N_{1}\right) \vee F\left(N_{2}\right)$ is the orbit partition of $N_{1} N_{2}$. Even if $N_{i}$ is the whole partwise stabilizer of $F\left(N_{i}\right)$ for $i=1,2$, the partwise stabilizer of $F\left(N_{1}\right) \vee F\left(N_{2}\right)$ may be larger than $N_{1} N_{2}$, so in general we do not insist on this condition.

Now the base group $B$ of the extended crested product is defined to be the group generated by the subgroups $\rho(F)^{F}$ for $F \in \mathcal{F}$. (As usual, $\rho(F)^{F}$ is embedded in $G_{2}^{\Omega_{1}}$ as the set of functions constant on parts of $F$ and taking values in $\rho(F)$.)

The extended crested product is generated by the base group and the group $G_{1}$ acting coordinatewise, as before. It is clear that each subgroup $\rho(F)^{F}$ is normalized by $G_{1}$, so we do have a semi-direct product; but the structure of the base group is less clear.

Example 7 . Let $\Omega_{1}$ be $\underline{\underline{2}} \times \underline{\underline{2}}$ and let $G_{2}$ be the dihedral group

$$
\left\langle a, b: a^{4}=b^{2}=1, b a b=a^{-1}\right\rangle
$$

acting on the corners of a square. Take $F_{1}$ and $F_{2}$ to be the partitions of $\Omega_{1}$ into rows and columns respectively, with $\rho\left(F_{1}\right)=\left\langle a^{2}, b\right\rangle$ and $\rho\left(F_{2}\right)=\left\langle a^{2}, a b\right\rangle$.

Figure 4 shows elements $\phi_{i}$ in $\rho\left(F_{i}\right)^{F_{i}}$ for $i=1,2$, and their product $\phi_{1} \phi_{2}$. It is readily checked that there are no elements $\phi_{i}^{\prime}$ in $\rho\left(F_{i}\right)^{F_{i}}$ for $i=1,2$ such that $\phi_{2}^{\prime} \phi_{1}^{\prime}=\phi_{1} \phi_{2}$. Hence these two subgroups do not commute, so their product is not a group.

$$
\phi_{1}=\begin{array}{|l|l|}
\hline 1 & 1 \\
\hline b & b \\
\hline
\end{array} \quad \phi_{2}=\begin{array}{|l|l|}
\hline 1 & a b \\
\hline 1 & a b \\
\hline
\end{array} \quad \phi_{1} \phi_{2}=\begin{array}{|l|l|}
\hline 1 & a b \\
\hline b & a^{3} \\
\hline
\end{array}
$$

FigURE 4. Non-commuting subgroups of the base group in Example 7

Nonetheless, we can indeed prove that the base group $B$ is the product, in a suitable order, of all the subgroups that generate it. We use the fact that every partial order can be embedded in a total order.

Theorem 12. Suppose that $|\mathcal{F}|=n$. Relabel the partitions in $\mathcal{F}$ by $1, \ldots, n$ such that $i \leqslant j$ whenever $F_{i} \preccurlyeq F_{j}$. Put $N_{i}=\rho\left(F_{i}\right)$ and $B_{i}=N_{i}^{F_{i}}$ for $i=1, \ldots, n$. Then $B_{1} B_{2} \ldots B_{n}$ is a group.

Proof. Assume for induction that

(a) $B_{1} B_{2} \ldots B_{i}$ is a group $C_{i}$;

(b) $C_{i}$ commutes with $B_{j}$ whenever $i<j \leqslant n$. 
These statements are both true when $i=0$, for then $C_{i}$ is the trivial group.

Assume that both are true for all non-negative integers less than $i$. Statement (b) for $i-1$ shows that $C_{i-1} B_{i}$ is a group, so that (a) is true for $i$.

Consider the product $C_{i} B_{j}$, where $i<j \leqslant n$. If $F_{i} \preccurlyeq F_{j}$ then $B_{j}$ normalizes $B_{i}$ so $B_{i} B_{j}=B_{j} B_{i}$. Otherwise, since $\mathcal{N}$ is meet-closed and $\rho$ is onto, there is some $k$ with $1 \leqslant k \leqslant i-1$ and $F_{k} \preccurlyeq F_{i} \wedge F_{j}$ and $\rho\left(F_{k}\right)=N_{i} \cap N_{j}$. If $\phi_{i} \in B_{i}$ and $\phi_{j} \in B_{j}$ then $\phi_{i}$ and $\phi_{j}$ take constant values (in $N_{i}$ and $N_{j}$ respectively) throughout each part of $F_{k}$, and these values commute modulo $N_{i} \cap N_{j}$, which is $N_{k}$. Hence $B_{k} B_{i} B_{j}=B_{k} B_{j} B_{i}$. Use of (b) for $i-2, i-3, \ldots, k$ shows that $C_{i-1}=B_{1} B_{2} \ldots B_{i-1}=B_{i-1} B_{i-2} \ldots B_{k+1} B_{1} B_{2} \ldots B_{k}$. Therefore

$$
\begin{aligned}
C_{i} B_{j}=C_{i-1} B_{i} B_{j} & =B_{i-1} B_{i-2} \ldots B_{k+1} B_{1} B_{2} \ldots B_{k} B_{i} B_{j} \\
& =B_{i-1} B_{i-2} \ldots B_{k+1} B_{1} B_{2} \ldots B_{k} B_{j} B_{i} \\
& =C_{i-1} B_{j} B_{i} .
\end{aligned}
$$

In both cases, $C_{i} B_{j}=C_{i-1} B_{j} B_{i}$, which is equal to $B_{j} C_{i-1} B_{i}$ by (b) for $i-1$, which is $B_{j} C_{i}$. Thus statement (b) is true for $i$.

Now it is easily checked that both crested products of groups mentioned in Section 7 are indeed produced by this construction: for the simple crested product $\mathcal{F}=\left\{F_{1}, U_{1}\right\}, \rho\left(F_{1}\right)=N$ and $\rho\left(U_{1}\right)=G_{2}$; while the 'alternative' crested product is obtained when $\mathcal{F}=\left\{E_{1}, F_{1}\right\}, \rho\left(E_{1}\right)=N$ and $\rho\left(F_{1}\right)=G_{2}$. Also, the extended crested product contains the direct product and is contained in the wreath product.

At first sight it seems strange that, for the group construction, $\mathcal{F}$ does not need to be closed under $\vee$ and $\wedge$. However, suppose that $F_{1}$ and $F_{2}$ are incomparable partitions in $\mathcal{F}$. Then $N_{i}^{F_{i}}$ contains $N_{i}^{F_{1} \vee F_{2}}$ for $i=1,2$ and so the base group contains $\left(N_{1} N_{2}\right)^{F_{1} \vee F_{2}}$. The orbits of $N_{1} \cap N_{2}$ may be smaller than the parts of $F\left(N_{1}\right) \wedge F\left(N_{2}\right)$ but there is some $k$ with $F_{k} \preccurlyeq F_{1} \wedge F_{2}$ and $N_{k}=N_{1} \cap N_{2}$, so the base group contains $\left(N_{1} \cap N_{2}\right)^{F_{k}}$, which contains $\left(N_{1} \cap N_{2}\right)^{F_{1} \wedge F_{2}}$. Thus including $F_{1} \vee F_{2}$ and $F_{1} \wedge F_{2}$ in $\mathcal{F}$ and putting $\rho\left(F_{1} \vee F_{2}\right)=N_{1} N_{2}$ and $\rho\left(F_{1} \wedge F_{2}\right)=N_{1} \cap N_{2}$ still satisfies all the conditions but adds nothing to the base group.

\section{Linking the groups to the schemes}

It is rather curious that the conditions on $\mathcal{F}$ and $\psi$ needed to ensure that the extended crested product of association schemes is indeed an association scheme are slightly different from those on $\mathcal{F}$ and $\rho$ which we require to ensure that the base group of the extended crested product of permutation groups is the product of its generating subgroups.

In order to link the extended crested product of permutation groups to the extended crested product of association schemes, we need to introduce an interesting operator on semi-lattices. If $\mathcal{F}$ is a meet-closed poset, define $F^{\perp}$, for $F$ in $\mathcal{F}$, to be the infimum of all those $K$ in $\mathcal{F}$ for which $K \npreceq F$. By convention, the empty infimum is the relevant maximal element, so that if $\mathcal{F}$ consists of partitions and $F$ is uniquely maximal in $\mathcal{F}$ then $F^{\perp}=U$, while if $\mathcal{F}$ consists of normal subgroups of a group $G$ and $F$ is uniquely maximal in $\mathcal{F}$ then $F^{\perp}=G$. The map $F \mapsto F^{\perp}$ preserves order and infima and has the property that, for any $F_{1}, F_{2}$ in $\mathcal{F}$, either $F_{1} \preccurlyeq F_{2}$ or $F_{2}^{\perp} \preccurlyeq F_{1}$. However, this map need not be one-to-one.

Dually, if $\mathcal{F}$ is join-closed then define $F^{\top}$, for $F$ in $\mathcal{F}$, to be the supremum of 
all those $K$ in $\mathcal{F}$ for which $K \nsucc F$. Then the map $F \mapsto F^{\top}$ preserves order and suprema, and either $F_{1} \preccurlyeq F_{2}$ or $F_{2} \preccurlyeq F_{1}^{\top}$.

Lemma 13. Let $\mathcal{F}$ be a lattice.

(a) If $F \in \mathcal{F}$ and $F \npreceq F^{\top}$ then $F^{\top \perp}=F$.

(b) If $\mathcal{F}$ is distributive and $F \in \mathcal{F}$ and $F$ is join-irreducible then $F \npreceq F^{\top}$.

Proof.

(a) For $K$ in $\mathcal{F}$, if $K \nprec F^{\top}$ then $F \preccurlyeq K$. Hence $F \preccurlyeq F^{\top \perp}$. If $F \npreceq F^{\top}$ then $F^{\top \perp} \preccurlyeq F$. Therefore $F^{\top \perp}=F$.

(b) By definition, $F^{\top}=\bigvee\{K: K \nsucc F\}$, so distributivity implies that $F \wedge F^{\top}=$ $\bigvee\{F \wedge K: K \nsucc F\}$. If $K \nsucc F$ then $F \wedge K \neq F$, so $\bigvee\{F \wedge K: K \nsucc F\}$ cannot be equal to $F$, because $F$ is join-irreducible. Hence $F \wedge F^{\top} \neq F$, and so $F \nprec F^{\top}$.

In order that the extended crested product of groups preserve the extended crested product of the association schemes, we need the sets of conditions for the two products to be defined, and a link between $\psi$ and $\rho$. We obtain the link by applying the operator ${ }^{\perp}$ to the set of normal subgroups of $G_{2}$ which are the partwise stabilizers of the partitions $\psi(F)$.

THEOREM 14. Suppose that $\mathcal{F}_{r}$ is a set of inherent partitions in the association scheme $\mathcal{Q}_{r}$ on $\Omega_{r}$, and that the permutation group $G_{r}$ preserves the classes of $\mathcal{Q}_{r}$, for $r=1$, 2. Suppose that $\mathcal{F}_{1}$ contains $U_{1}$ and is closed under $\wedge$ and $\vee$, and that $\psi$ is a map from $\mathcal{F}_{1}$ to $\mathcal{F}_{2}$ which preserves order and suprema and which carries the unique minimal element $F_{0}$ of $\mathcal{F}_{1}$ to $E_{2}$. For $F$ in $\mathcal{F}_{1}$, suppose that there exists a normal subgroup $N_{\psi(F)}$ of $G_{2}$ whose orbit partition is $\psi(F)$, and let $\rho(F)$ be the intersection of the $N_{\psi(H)}$ for $H$ in $\mathcal{F}_{1}$ with $H \npreceq F$. Then the crested product of $G_{1}$ and $G_{2}$ with respect to $\rho$ preserves the classes of the crested product of $\mathcal{Q}_{1}$ and $\mathcal{Q}_{2}$ with respect to $\psi$.

Proof. Put $\mathcal{N}=\rho\left(\mathcal{F}_{1}\right)$. Then $G_{2}=\rho\left(U_{1}\right) \in \mathcal{N}$ and $\rho(F) \cap \rho(H)=\rho(F \wedge H)$ for $F, H$ in $\mathcal{F}_{1}$, so $\rho$ satisfies the conditions to define the crested product of permutation groups.

The argument for preserving the association scheme is similar to that for the simple crested product. Suppose that the pairs $\left(\alpha_{1}, \alpha_{2}\right)$ and $\left(\beta_{1}, \beta_{2}\right)$ satisfy the relation with matrix $A_{i} \otimes D$, where $i \in \mathcal{J}_{F}$ and $D \in \mathcal{D}_{F}$, and take $\phi$ in $(\rho(H))^{H}$; we must show that $\left(\alpha_{1}, \alpha_{2} \phi\left(\alpha_{1}\right)\right)$ and $\left(\beta_{1}, \beta_{2} \phi\left(\beta_{1}\right)\right)$ satisfy the same relation.

If $F \preccurlyeq H$, then $\phi\left(\alpha_{1}\right)=\phi\left(\beta_{1}\right)$, which is in $G_{2}$ and preserves the $D$-relation. On the other hand, if $F \npreceq H$, then $\rho(H)$ stabilizes $\psi(F)$ partwise; however, the $D$-relation depends only on the $\psi(F)$-classes.

We now consider some examples.

In the case where $\mathcal{F}_{1}$ is a chain, so that $\rho(F)$ is the partwise stabilizer of the partition immediately above $\psi(F)$ for $F \neq U$, we see that if $F_{1} \preccurlyeq F_{2}$ then $\left(\rho\left(F_{2}\right)\right)^{F_{2}}$ normalizes $\left(\rho\left(F_{1}\right)\right)^{F_{1}}$, so the base group is their product. We have seen that the simple crested product and the modified version in Section 7 are examples. 
EXAMPLE 8. In these examples, we assume that the set of partitions in each of $\mathcal{Q}_{1}$ and $\mathcal{Q}_{2}$ satisfy the appropriate order and meet relations in one of the diagrams in Figure 5. Moreover, we have partitions $F_{i}$ for each label $i$ in the diagram for $\mathcal{Q}_{1}$, partitions $K_{i}$ for each label $i$ in the diagram for $\mathcal{Q}_{2}$, and $N_{i}$ is a normal subgroup of $G_{2}$ whose orbit partition is $K_{i}$. We also assume that $K_{1}=E_{1}$ in each case, so that $N_{1}=1$, and that the coarsest partition shown is $U$.

First, take $\mathcal{Q}_{1}$ and $\mathcal{Q}_{2}$ both to have the inherent partitions in Figure $5(\mathrm{a})$, with the obvious isomorphism $\psi$. Then $\rho\left(F_{i}\right)=N_{i}^{\perp}=N_{1}$ for all $i \neq 5$, while $N_{5}^{\perp}=G_{2}$. Therefore the extended crested product of the groups is just the direct product, even though the extended crested product of the association schemes is not.

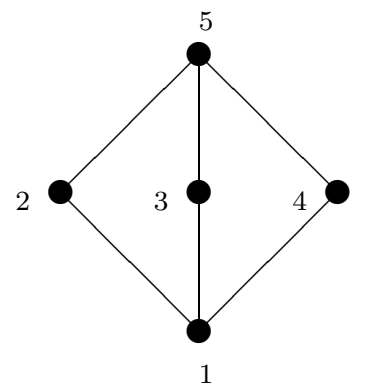

FiguRE 5. Examples of extended crested products of groups

Secondly, we can take $\mathcal{Q}_{1}$ and $\mathcal{Q}_{2}$ to be as in Figures $5(\mathrm{~b})$ and (a) respectively, with $\psi\left(F_{i}\right)=K_{i}$ for $i=1,2,3,4$ and $\psi\left(F_{i}\right)=U$ otherwise. Then the base group of the extended crested product of the groups is just the product of $G_{2}^{F_{5}}, G_{2}^{F_{6}}$ and $G_{2}^{F_{7}}$ : the partitions $F_{2}, F_{3}, F_{4}, K_{2}, K_{3}$, and $K_{4}$ contribute nothing to the group even though they play a role in the association scheme.

If we reverse the roles of $\mathcal{Q}_{1}$ and $\mathcal{Q}_{2}$ then we can take $\psi\left(F_{i}\right)=K_{i+3}$ for $i=2$, 3,4 and find that the base group of the extended crested product of the groups is generated by $\left(N_{6} \cap N_{7}\right)^{F_{2}},\left(N_{5} \cap N_{7}\right)^{F_{3}},\left(N_{5} \cap N_{6}\right)^{F_{4}}$ and $G_{2}^{U}$; the subgroups $N_{5}$, $N_{6}$ and $N_{7}$ do not appear in the expression for the base group.

However, if we take both $\mathcal{Q}_{1}$ and $\mathcal{Q}_{2}$ to be as in Figure $5(\mathrm{~b})$, with the obvious isomorphism $\psi$, then, as we shall show in the next theorem, the group and the association scheme match perfectly, and we obtain a generalization of Theorem 11 .

THEOREM 15. Suppose that, in addition to the hypotheses of Theorem 14, the following hold:

(i) $\mathcal{F}$ is distributive,

(ii) $\psi$ is a lattice isomorphism,

(iii) $N_{\psi\left(F_{1}\right)} \cap N_{\psi\left(F_{2}\right)}=N_{\psi\left(F_{1}\right) \wedge \psi\left(F_{2}\right)}$ and $N_{\psi\left(F_{1}\right)} N_{\psi\left(F_{2}\right)}=N_{\psi\left(F_{1}\right) \vee \psi\left(F_{2}\right)}$, for all $F_{1}$ and $F_{2}$ in $\mathcal{F}$, and

(iv) the orbits of $G_{r}$ on $\Omega_{1} \times \Omega_{2}$ are the classes of $\mathcal{Q}_{r}$ for $r=1,2$.

Then the orbits of $G$ on $\Omega \times \Omega$ are the classes of $\mathcal{Q}$. 
Proof. Since we have already shown that $G$ preserves the classes of $\mathcal{Q}$, all we need to do is show that each class of $\mathcal{Q}$ is a single orbit of $G$ on $\Omega \times \Omega$.

Consider the class with adjacency matrix $A_{i} \otimes D$, where $i \in \mathcal{J}_{F}$ and $D \in \mathcal{D}_{F}$ for some $F$ in $\mathcal{F}$. Suppose that $\left(\left(\alpha_{1}, \beta_{1}\right),\left(\gamma_{1}, \delta_{1}\right)\right)$ and $\left(\left(\alpha_{2}, \beta_{2}\right),\left(\gamma_{2}, \delta_{2}\right)\right)$ are both in this class. There is an element of the top group which takes $\alpha_{1}$ to $\alpha_{2}$ and $\gamma_{1}$ to $\gamma_{2}$, so we may suppose that $\alpha_{1}=\alpha_{2}=\alpha$ and $\gamma_{1}=\gamma_{2}=\gamma$.

If $F$ is the minimal element of $\mathcal{F}$ then $\psi(F)=E_{2}$ so $D$ is just an adjacency matrix for $\mathcal{Q}_{2}$ and so there is some $g$ in $G_{2}$ with $\left(\beta_{1}, \delta_{1}\right) g=\left(\beta_{2}, \delta_{2}\right)$.

If $F$ is not the minimal element of $\mathcal{F}$ then there is a positive integer $n$ and join-irreducibles $H_{1}, \ldots, H_{n}$ in $\mathcal{F}$ such that $F=H_{1} \vee \ldots \vee H_{n}$. For $m=1$, $\ldots, n$, Lemma 13(b) shows that $H_{m} \npreceq H_{m}^{\top}$. Write $N_{m}$ for $N_{\psi\left(H_{m}\right)}$. Because $\psi$ and the map $\psi(K) \mapsto N_{\psi(K)}$ are both lattice isomorphisms, Lemma 13(a) shows that $\rho\left(H_{m}^{\top}\right)=N_{m}$, so that the base group contains $N_{m}^{H_{m}^{\top}}$.

Since $\left(\beta_{1}, \delta_{1}\right)$ and $\left(\beta_{2}, \delta_{2}\right)$ are both in the $D$-relation, there is some $j$ in $\mathcal{K}_{2}$ and points $\varepsilon_{1}, \varepsilon_{2}$ in $\Omega_{2}$ such that $\left(\beta_{1}, \varepsilon_{1}\right)$ and $\left(\beta_{2}, \varepsilon_{2}\right)$ are in the $B_{j}$-class of $\mathcal{Q}_{2}$ while $\varepsilon_{r}$ is in the same part of $\psi(F)$ as $\delta_{r}$ for $r=1,2$. Now, $\psi(F)=\psi\left(H_{1}\right) \vee \ldots \vee \psi\left(H_{n}\right)$, and the matrices $R_{\psi\left(H_{m}\right)}$ commute pairwise, so there are elements $\zeta_{r 0}, \zeta_{r 1}, \ldots, \zeta_{r n}$ in $\Omega_{2}$, for $r=1,2$, such that $\varepsilon_{r}=\zeta_{r 0}, \delta_{r}=\zeta_{r n}$, and $\zeta_{r, m-1}$ and $\zeta_{r, m}$ are in the same part of $\psi\left(H_{m}\right)$ for $m=1, \ldots, n$.

The extended crested product $G$ of the groups contains $G_{2}$ acting diagonally, so it contains an element $\phi_{0}$ taking $\left(\beta_{1}, \varepsilon_{1}\right)$ to $\left(\beta_{2}, \varepsilon_{2}\right)$.

If $\alpha$ and $\gamma$ are in the same part of $H_{m}^{\top}$, then the definition of $\mathcal{J}_{F}$ shows that $H_{m}^{\top} \succcurlyeq F \succcurlyeq H_{m}$, which contradicts Lemma 13(b) for $H_{m}$. Hence $\alpha$ and $\gamma$ are in different parts of $H_{m}^{\top}$ for $m=1, \ldots, n$. For $r=1,2$ and $m=1, \ldots, n$ there is an element $g_{r m}$ in $N_{m}$ such that $\zeta_{r, m-1} g_{r m}=\zeta_{r, m}$. Hence there are elements $\phi_{1 m}$ and $\phi_{2 m}$ in $N_{m}^{H_{m}^{\top}}$ such that $\phi_{1 m}(\alpha)$ and $\phi_{2 m}(\alpha)$ are the identity, $\phi_{1 m}(\gamma)=g_{1 m}^{-1}$ and $\phi_{2 m}(\gamma)=g_{2 m}$ for $m=1, \ldots, n$. Now $\beta_{1} \phi_{1 n}(\alpha) \ldots \phi_{11}(\alpha) \phi_{0} \phi_{21}(\alpha) \ldots \phi_{2 n}(\alpha)=\beta_{2}$ and $\delta_{1} \phi_{1 n}(\gamma) \ldots \phi_{11}(\gamma) \phi_{0} \phi_{21}(\gamma) \ldots \phi_{2 n}(\gamma)=\delta_{2}$.

\section{Remarks and problems}

We have not attempted to calculate the character table of a generalized crested product, or to give conditions for the generalized crested product to have a formal dual.

Another generalization of direct and wreath product in the literature is that of the generalized wreath product $[\mathbf{4}, \mathbf{5}]$. We do not define this here; but note that it is a product of a family of association schemes (or permutation groups) indexed by a partially ordered set, that it reduces to direct or wreath product in case the partially ordered set is a two-element antichain or chain respectively, and that the generalized wreath product of trivial association schemes is the scheme derived from a poset block structure (using the same poset).

One could now try to combine the two constructions. There are two possible ways in which this combination could take place. First, we could take the (extended) crested product of generalized wreath products. As noted above, a generalized wreath product of trivial schemes is a poset block structure, and we have already seen that a crested product of poset block structures is a poset block structure (so at least in this case we obtain nothing new). 
The other possible combination is far more speculative. Thinking of a generalized wreath product as built from the ingredients "direct product" and "wreath product", applied to schemes whose indices are incomparable or comparable respectively, we could try to replace these ingredients by the more general notion of crested product. It is not even clear what kind of mathematical structure should replace the poset on the index set of the association schemes in order to describe such a construction!

An important question we have not considered is that of counting orbits of permutation groups on $n$-tuples of points (or more generally, of calculating the cycle index of permutation groups). We refer to [10] for the cycle index of the direct and wreath products (the first of these is, of course, well known). Also, in the paper [16], the number of orbits on $n$-tuples of a generalized wreath product of symmetric groups is calculated. There are many open problems here.

\section{References}

1. R. A. BAILEY, 'Factorial design and Abelian groups', Linear Algebra and its Applications 70 (1985) 349-368.

2. R. A. BAiley, 'Orthogonal partitions in designed experiments', Designs, Codes and Cryptography 8 (1996) 45-77.

3. R. A. BAILEy, 'Suprema and infima of association schemes', Discrete Mathematics 248 (2002) $1-16$.

4. R. A. BAILEY, 'Generalized wreath products of association schemes', preprint, 2003.

5. R. A. Bailey, Cheryl E. Praeger, C. A. Rowley and T. P. Speed, 'Generalized wreath products of permutation groups', Proc. London Math. Soc., Series 347 (1983) 69-82.

6. E. BAnnai, 'An introduction to association schemes', Methods of Discrete Mathematics (eds. S. Löwe, F. Mazzocca, N. Melone and U. Ott), Quaderni di Mathematica, 5, (Dipartimento di Matematica della Seconda Università di Napoli, Napoli, 1999) 1-70.

7. E. Bannai and T. Ito, Algebraic Combinatorics. I: Association Schemes, (Benjamin, Menlo Park, California, 1984).

8. R. C. Bose And T. Shimamoto, 'Classification and analysis of partially balanced incomplete block designs with two associate classes', Journal of the American Statistical Association 47 (1952) 151-184.

9. W. G. Bridges and R. A. Mena, 'Rational circulants with rational spectra and cyclic strongly regular graphs', Ars Combinatorica 8 (1979) 143-161.

10. Peter J. Cameron, Daniele A. Gewurz and Francesca Merola, 'Product action', preprint, 2003.

11. P. J. Cameron, J.-M. Goethals and J. J. Seidel, 'The Krein condition, spherical designs, Norton algebras and permutation groups', Proceedings of the Koninklijke Nederlandse Akademie van Wetenschappen, Series A 81 (1978) 196-206.

12. P. J. Cameron and J. H. van Lint, Designs, Graphs, Codes and their Links, London Mathematical Society Student Texts, 22, (Cambridge University Press, Cambridge, 1991).

13. P. Delsarte, An algebraic approach to the association schemes of coding theory, Ph. D. thesis, Université Catholique de Louvain, 1973. (appeared as Philips Research Reports Supplement, No. 10, 1973)

14. D. G. Higman, 'Coherent configurations I', Geometriae Dedicata 4 (1975) 1-32.

15. J. A. Nelder, 'The analysis of randomized experiments with orthogonal block structure. I. Block structure and the null analysis of variance', Proceedings of the Royal Society of London, Series A 283 (1965) 147-162.

16. Cheryl E. Praeger, C. A. Rowley and T. P. Speed, 'A note on generalised wreath products', J. Austral. Math. Soc., Series A 39 (1985) 415-420.

17. T. P. SPEED AND R. A. BAILey, 'On a class of association schemes derived from lattices of equivalence relations', Algebraic Structures and Applications (eds. P. Schultz, C. E. Praeger and R. P. Sullivan), (Marcel Dekker, New York, 1982) 55-74.

18. M. C. Whelan, Imprimitive association schemes, Ph. D. thesis, University of London. 1989. 
R. A. Bailey and Peter J. Cameron,

School of Mathematical Sciences

Queen Mary, University of London,

Mile End Road, London, E1 $4 N S$

r.a.bailey@qmul.ac.ak

p.j.cameron@qmul.ac.uk 